QUARTERLY OF APPLIED MATHEMATICS

VOLUME LXIX, NUMBER 2

JUNE 2011, PAGES 331-356

S 0033-569X(2011)01241-5

Article electronically published on March 9, 2011

\title{
GLOBAL REGULARITY \\ FOR A COUPLED CAHN-HILLIARD-BOUSSINESQ SYSTEM ON BOUNDED DOMAINS
}

\author{
BY \\ KUN ZHAO \\ Mathematical Biosciences Institute, Ohio State University, Columbus, Ohio 43210
}

\begin{abstract}
We study an initial-boundary value problem (IBVP) for a coupling of the Cahn-Hilliard equation with the 2D inviscid heat-conductive Boussinesq equations. For large initial data with finite energy, we prove global existence and uniqueness of classical solutions to the IBVP, together with some uniform-in-time and decay estimates of the solution.
\end{abstract}

1. Introduction. One major challenge in mathematical fluid dynamics is the questions of global existence, uniqueness and large time asymptotic behavior of classical solutions to the Cauchy problem or initial-boundary value problems for modeling equations. Not only are the questions physically important, they are also mathematically challenging. Positive answers to these questions will undoubtedly benefit both mathematicians and engineers. In the real world, flows often move in bounded domains with constraints from boundaries, where initial-boundary value problems (IBVP) appear. Solutions to initial-boundary value problems usually exhibit different behaviors and much richer phenomena compared with the Cauchy problem.

In this paper, we consider the following system of equations:

$$
\left\{\begin{array}{l}
\phi_{t}+U \cdot \nabla \phi=\Delta \mu \\
\mu=-\alpha \Delta \phi+F^{\prime}(\phi), \\
U_{t}+U \cdot \nabla U+\nabla P=\mu \nabla \phi+\theta \mathbf{e}_{2}, \\
\theta_{t}+U \cdot \nabla \theta=\kappa \Delta \theta \\
\nabla \cdot U=0
\end{array}\right.
$$

which is obtained by coupling the famous Cahn-Hilliard equation:

$$
\phi_{t}=\Delta\left(-\alpha \Delta \phi+F^{\prime}(\phi)\right)
$$

Received October 23, 2009.

2010 Mathematics Subject Classification. Primary 35Q35, 35B65, 35B40.

Key words and phrases. Cahn-Hilliard equation, 2D Boussinesq equations, global existence and uniqueness.

E-mail address: kzhao@mbi.ohio-state.edu. 
with the $2 \mathrm{D}$ inviscid heat-conductive Boussinesq equations:

$$
\left\{\begin{array}{l}
U_{t}+U \cdot \nabla U+\nabla P=\theta \mathbf{e}_{2} \\
\theta_{t}+U \cdot \nabla \theta=\kappa \Delta \theta \\
\nabla \cdot U=0
\end{array}\right.
$$

through convection and order parameters. System (1.1) describes the motion of an incompressible inviscid two-phase flow subject to convective heat transfer under the influence of gravitational force. Here, $\phi$ is the order parameter and $\mu$ is a chemical potential derived from a coarse-grained study of the free energy of the fluid (cf. [16]), $U=(u, v), P$ and $\theta$ denote the velocity, pressure and temperature respectively; the constants $\alpha>0$ and $\kappa>0$ model diffusion and heat conduction respectively, and $\mathbf{e}_{2}=(0,1)^{\mathrm{T}}$.

Our work in the current paper is motivated, first, by [6], in which the coupling of the Cahn-Hilliard equation with the Navier-Stokes equations was considered:

$$
\left\{\begin{array}{l}
\phi_{t}+U \cdot \nabla \phi=\Delta \mu, \\
\mu=-\alpha \Delta \phi+F^{\prime}(\phi), \\
U_{t}+U \cdot \nabla U+\nabla P=\nu \Delta U+\mu \nabla \phi, \\
\nabla \cdot U=0,
\end{array}\right.
$$

which describes the motion of an incompressible two-phase flow under shear through order parameter formulation. In [6], the author considered (1.4) in a channel $\Omega=$ $(-L, L)^{d-1} \times(-1,1)$, where $d=2$ or 3 , and proved global (local resp.) well-posedness of strong solutions to the IBVP in $\mathbb{R}^{2}$ ( $\mathbb{R}^{3}$ resp.). The boundary conditions considered in [6] are as follows:

$$
\left\{\begin{array}{l}
\nabla \phi \cdot \mathbf{n}=\nabla \mu \cdot \mathbf{n}=0 \text { on }\{z= \pm 1\}, \\
U=\bar{U} \mathbf{e}_{1} \text { on }\{z=1\} \text { and } U=-\bar{U} \mathbf{e}_{1} \text { on }\{z=-1\}, \\
\phi \text { and } U \text { are } 2 L \text {-periodic }\left(L \text {-periodic in } \mathbb{R}^{2}\right) \text { in the variables } x \text { and } y\left(x \text { in } \mathbb{R}^{2}\right),
\end{array}\right.
$$

where $\mathbf{n}$ is the unit outward normal to $\partial \Omega, \bar{U}>0$ is a constant and $\mathbf{e}_{1}$ is the first unit vector. In addition to the global existence results, the asymptotic behavior of weak solutions to the IBVP was also investigated in the paper. However, we note that the global existence results obtained in [6] ceased at the level of strong solutions, and the smoothness of the solution was not discussed there. We refer the readers to [7, 8] and the references therein for more discussions in this direction. For more studies on two-phase flow, we refer the readers to $33,4,10,13,14,15,17,28,29,31]$ and the references therein.

The second motivation of our work in this paper comes from the studies of the wellknown 2D Boussinesq system:

$$
\left\{\begin{array}{l}
U_{t}+U \cdot \nabla U+\nabla P=\nu \Delta U+\theta \mathbf{e}_{2} \\
\theta_{t}+U \cdot \nabla \theta=\kappa \Delta \theta \\
\nabla \cdot U=0
\end{array}\right.
$$

which is closely related to the studies of 3D incompressible flows (cf. 27, 30. ) and has been well studied in the literature. The readers are referred to [9, 11, 12, 18, 19] and the references therein for the Cauchy problem, and to [22, 26, 32] and the references therein 
for initial-boundary value problems, where global existence and large time behavior of solutions to 2D Boussinesq equations with full or partial viscosity terms are investigated. Especially, in 32, the author considered the initial-boundary value problem for 2D inviscid heat-conductive Boussinesq equations:

$$
\left\{\begin{array}{l}
(1.3) ; \\
(U, \theta)(\mathbf{x}, 0)=\left(U_{0}, \theta_{0}\right)(\mathbf{x}), \\
\left.U \cdot \mathbf{n}\right|_{\partial \Omega}=0,\left.\theta\right|_{\partial \Omega}=\bar{\theta}
\end{array}\right.
$$

where $\Omega \subset \mathbb{R}^{2}$ is a bounded domain with smooth boundary and $\bar{\theta}$ is a constant, and proved that, for given large initial data with finite energy satisfying certain compatibility conditions, there exists a unique global in time classical solution to (1.6). Moreover, it is shown that the temperature $\theta$ converges to $\bar{\theta}$ exponentially fast as time goes to infinity and the velocity $U$ and the vorticity $\omega=v_{x}-u_{y}$ are uniformly bounded in time in certain topologies.

In this paper, following [6] and [32, we consider the coupled system (1.1) in a bounded domain in $\mathbb{R}^{2}$. The system is supplemented by the following initial and boundary conditions:

$$
\left\{\begin{array}{l}
(\phi, \mu, U, \theta)(\mathbf{x}, 0)=\left(\phi_{0}, \mu_{0}, U_{0}, \theta_{0}\right)(\mathbf{x}) \\
\left.\nabla \phi \cdot \mathbf{n}\right|_{\partial \Omega}=0,\left.\nabla \mu \cdot \mathbf{n}\right|_{\partial \Omega}=0,\left.U \cdot \mathbf{n}\right|_{\partial \Omega}=0,\left.\theta\right|_{\partial \Omega}=\bar{\theta}
\end{array}\right.
$$

where $\Omega \subset \mathbb{R}^{2}$ is a bounded domain with smooth boundary $\partial \Omega, \mathbf{n}$ is the unit outward normal to $\partial \Omega$ and $\bar{\theta}$ is a constant.

We remark here that one of the major challenges in dealing with the global regularity of (1.3) is the loss of viscosity in the velocity equation which leads to the fact that the regularity of $U$ can be built up only after the $H^{3}$ estimate of $\theta$ is established according to the classical results on 2D incompressible nonhomogeneous Euler equations (cf. 20, 23]). Due to the coupling of the velocity equation with the temperature equation by nonlinear convection and gravitational force, the $H^{3}$ estimate of $\theta$ is a substantial barrier to overcome. Taking this consideration into (1.1) we see that the complexity of the analysis of (1.1) will significantly increase due to the further coupling of the CahnHilliard equation. On the other hand, we note that, for (1.1), another challenge brought by the loss of viscosity is the treatment of the regularity of the order parameter $\phi$, which greatly enriches the analysis of (1.1) compared with (1.4). To the best of the author's knowledge, the initial-boundary value problem (1.1), (1.7) has not been studied in the literature 1 In the current paper, we generalize the studies of $[6$ and 32 to the case of two-phase flow without shear but subject to convective heat transfer and under the influence of gravitational force. We prove global existence and uniqueness of classical solutions to the initial-boundary value problem (1.1), (1.7) for large initial data with finite energy.

Throughout this paper, $\|\cdot\|_{L^{p}},\|\cdot\|_{L^{\infty}}$ and $\|\cdot\|_{W^{s, p}}$ denote the norm of the usual Lebesgue measurable function spaces $L^{p}(1 \leq p<\infty), L^{\infty}$ and the usual Sobolev space

\footnotetext{
${ }^{1}$ The global existence and uniqueness of classical solutions to an IBVP for the coupling of (1.2) with (1.5) with $\nu>0$ and $\kappa=0$ were established recently in 33 .
} 
$W^{s, p}$ respectively. For $p=2$, we denote the norm $\|\cdot\|_{L^{2}}$ by $\|\cdot\|$ and $\|\cdot\|_{W^{s, 2}}$ by $\|\cdot\|_{H^{s}}$ respectively. The function spaces under consideration are

$$
C\left([0, T] ; H^{r}(\Omega)\right) \text { and } L^{2}\left([0, T] ; H^{s}(\Omega)\right),
$$

equipped with norms

$$
\sup _{0 \leq t \leq T}\|\Psi(\cdot, t)\|_{H^{r}} \text { and }\left(\int_{0}^{T}\|\Psi(\cdot, \tau)\|_{H^{s}}^{2} d \tau\right)^{1 / 2},
$$

where $r, s$ are positive integers. Throughout this paper, unless specified otherwise, $C$ will denote a generic constant which is independent of the unknown functions $\phi, \mu, U, \theta$, but may depend on $\alpha, \kappa, \Omega$, initial data and the time $T$. The value of $C$ may vary line by line according to the context.

For global existence of classical solutions to (1.1), (1.7), we require the following compatibility conditions:

$$
\left\{\begin{array}{l}
\nabla \cdot U_{0}=0, \mu_{0}=-\alpha \Delta \phi_{0}+F^{\prime}\left(\phi_{0}\right), \quad \mathbf{x} \in \Omega, \\
\left.\nabla \phi_{0} \cdot \mathbf{n}\right|_{\partial \Omega}=0,\left.\nabla \mu_{0} \cdot \mathbf{n}\right|_{\partial \Omega}=0,\left.U_{0} \cdot \mathbf{n}\right|_{\partial \Omega}=0,\left.\theta_{0}\right|_{\partial \Omega}=\bar{\theta}, \\
\left.\nabla\left(U_{0} \cdot \nabla \phi_{0}-\Delta \mu_{0}\right) \cdot \mathbf{n}\right|_{\partial \Omega}=0, \\
\left.\left(U_{0} \cdot \nabla \theta_{0}-\kappa \Delta \theta_{0}\right)\right|_{\partial \Omega}=0 .
\end{array}\right.
$$

The following theorem is the main result of this paper.

THEOREM 1.1. Let $\Omega \subset \mathbb{R}^{2}$ be a bounded domain with smooth boundary. Suppose that $F(\cdot)$ satisfies the following conditions:

$\left(H_{1}\right) \quad F(\cdot)$ is of $C^{6}$ class and $F(\cdot) \geq 0$.

$\left(H_{2}\right)$ There exist constants $F_{1}, F_{2}>0$ such that $\left|F^{(n)}(\phi)\right| \leq F_{1}|\phi|^{p-n}+F_{2}$, $n=1, \ldots, 6, \forall 6 \leq p<\infty$ and $\phi \in \mathbb{R}$.

$\left(H_{3}\right)$ There exists a constant $F_{3} \geq 0$ such that $F^{\prime \prime} \geq-F_{3}$.

If $\phi_{0}(\mathbf{x}) \in H^{5}(\Omega), \mu_{0}(\mathbf{x}) \in H^{3}(\Omega)$ and $\left(\theta_{0}(\mathbf{x}), U_{0}(\mathbf{x})\right) \in H^{3}(\Omega)$ satisfy the compatibility conditions (1.8), then there exists a unique solution $(\phi, \mu, \theta, U)$ to (1.1), (1.7) globally in time such that $\phi \in C\left([0, T] ; H^{5}(\Omega)\right) \cap L^{2}\left([0, T] ; H^{7}(\Omega)\right), \mu \in C\left([0, T] ; H^{3}(\Omega)\right) \cap$ $L^{2}\left([0, T] ; H^{5}(\Omega)\right), U \in C\left([0, T] ; H^{3}(\Omega)\right)$ and $\theta \in C\left([0, T] ; H^{3}(\Omega)\right) \cap L^{2}\left([0, T] ; H^{4}(\Omega)\right)$ for any $0<T<\infty$. Moreover, there exist constants $\gamma_{0}>0$ and $\eta_{0}(p) \geq 0$ which are independent of $t$ such that for any $t \geq 0$ and $p \in[2, \infty]$, it follows that

$$
\begin{gathered}
\|U(\cdot, t)\|^{2}+\|\phi(\cdot, t)\|_{H^{1}}^{2}+\|F(\phi(\cdot, t))\|_{L^{1}}+\int_{0}^{t}\|\nabla \mu(\cdot, \tau)\|^{2} d \tau \leq \gamma_{0}, \\
\|(\theta-\bar{\theta})(\cdot, t)\|_{L^{p}} \leq\left\|\theta_{0}-\bar{\theta}\right\|_{L^{p}} \exp \left\{-\eta_{0}(p) t\right\},
\end{gathered}
$$

where the constant $\eta_{0}(p)>0$ for $p \in[2, \infty)$ and $\eta_{0}(p)=0$ for $p=\infty$.

REMARK 1.1. The assumptions $\left(H_{1}\right)-\left(H_{3}\right)$ in Theorem 1.1 are similar to those in 6 ] and are satisfied for a number of important applications such as $F(x)=\left(1-x^{2}\right)^{2}$; see 6 and the references therein. It should be pointed out that, in the results obtained in Theorem 1.1, no smallness restriction is put upon the initial data. The results suggest 
that, without viscous dissipation, heat conduction and diffusion of the order parameter are still strong enough to prevent the development of a singularity in the system.

The proof of Theorem 1.1 begins with the global existence of weak solutions to (1.1), (1.7). This part can be proved by standard arguments, for example, the combination of the Galerkin approximation and the method of energy estimate (cf. [6]). In order to simplify the presentation, we shall not go through the details of the construction of the approximate solutions. Instead, we will focus on establishing the a priori estimates of the solution, which is essential for the global existence of classical solutions, in the major part of the paper. The energy estimate is somewhat delicate mainly due to the coupling between the Cahn-Hilliard equation and the Boussinesq equations by nonlinear convection, order parameter, gravitational force and boundary effects. Great efforts have been made to simplify the proof. There are intensive applications of Sobolev and Ladyzhenskaya type inequalities in the proof. The results on elliptic equations, see Lemma 2.2, are important in our energy framework. Roughly speaking, because of the lack of the spatial derivatives of the solution at the boundary, our energy framework proceeds as follows: We first apply the standard energy estimate on the solution and the temporal derivatives of the solution. We then apply the classical results on elliptic equations to obtain the spatial derivatives. Such a process will be repeated up to third order, and then the carefully coupled estimates will be composed into a desired estimate leading to global regularities of $\phi, \mu$ and $\theta$. After the $H^{3}$ estimate of the nonhomogeneous term on the right-hand side of the velocity equation is established, classical results on $2 \mathrm{D}$ incompressible nonhomogeneous Euler equations by Kato [20, see Lemma 2.4, will be implemented to build up the $H^{3}$ regularity of $U$. The uniform estimates of the solution stated in Theorem 1.1 are obtained by careful exploration of the structure of the system and delicate applications of the Cauchy-Schwarz inequality. The uniqueness is proved in a straightforward way by using the regularity of the solution.

The rest of this paper is organized as follows. In Section 2, we give some basic facts that will be used in this paper. Then we prove Theorem 1.1 and give some concluding remarks in Section 3.

2. Preliminaries. In this section, we shall collect several facts which will be used in the proof of Theorem 1.1. First, for the convenience of the readers, we recall some inequalities of Sobolev and Ladyzhenskaya type (cf. [1, 21]).

Lemma 2.1. Let $\Omega \subset \mathbb{R}^{2}$ be any bounded domain with smooth boundary $\partial \Omega$. Then

(i) $\|f\|_{L^{\infty}} \leq C\|f\|_{H^{2}}$;

(ii) $\|f\|_{L^{\infty}} \leq C\|f\|_{W^{1, p}}, \quad \forall p>2$;

(iii) $\|f\|_{L^{p}} \leq C\|f\|_{H^{1}}, \quad \forall 1 \leq p<\infty$;

(iv) $\|f\|_{L^{4}}^{2} \leq C\left(\|f\|\|\nabla f\|+\|f\|^{2}\right), \quad \forall f \in H^{1}(\Omega)$;

(v) $\|f\|_{L^{8}}^{2} \leq C\left(\|f\|\|\nabla f\|_{L^{4}}+\|f\|^{2}\right), \quad \forall f \in H^{1}(\Omega)$,

for some constants $C=C(p, \Omega)$.

Next, we recall some classical results on elliptic equations (cf. [2, 24, 25]). 
Lemma 2.2. Let $\Omega \subset \mathbb{R}^{2}$ be any bounded domain with smooth boundary $\partial \Omega$. Consider the Dirichlet problem:

$$
\left\{\begin{array}{l}
\kappa \Delta \Theta=f \text { in } \Omega, \\
\Theta=0 \text { on } \partial \Omega .
\end{array}\right.
$$

If $f \in W^{m, p}$, then $\Theta \in W^{m+2, p}$ and there exists a constant $C=C(p, \kappa, m, \Omega)$ such that

$$
\|\Theta\|_{W^{m+2, p}} \leq C\|f\|_{W^{m, p}}
$$

for any $p \in(1, \infty)$ and the integer $m \geq-1$.

Concerning the estimate of vector-valued functions, we have (see [5] )

Lemma 2.3. Let $\Omega \subset \mathbb{R}^{2}$ be any bounded domain with smooth boundary $\partial \Omega$, and let $F \in W^{s, p}(\Omega)$ be a vector-valued function satisfying $\left.F \cdot \mathbf{n}\right|_{\partial \Omega}=0$, where $\mathbf{n}$ is the unit outward normal to $\partial \Omega$. Then there exists a constant $C=C(s, p, \Omega)$ such that

$$
\|F\|_{W^{s, p}} \leq C\left(\|\nabla \times F\|_{W^{s-1, p}}+\|\nabla \cdot F\|_{W^{s-1, p}}+\|F\|_{L^{p}}\right)
$$

for any $s \geq 1$ and $p \in(1, \infty)$.

Now, we recall a general result for the 2D incompressible nonhomogeneous Euler equations on bounded domains with slip boundary condition (cf. [20, 23]).

Lemma 2.4. Let $\Omega \subset \mathbb{R}^{2}$ be any bounded domain with smooth boundary $\partial \Omega$. Consider the initial-boundary value problem:

$$
\left\{\begin{array}{l}
U_{t}+U \cdot \nabla U+\nabla P=G \\
\nabla \cdot U=0 \\
U(\mathbf{x}, 0)=U_{0}(\mathbf{x}),\left.\quad U \cdot \mathbf{n}\right|_{\partial \Omega}=0
\end{array}\right.
$$

where $\mathbf{n}$ is the unit outward normal to $\partial \Omega$. Let $U_{0}(\mathbf{x}) \in H^{3}(\Omega), \nabla \cdot U_{0}=0,\left.U_{0} \cdot \mathbf{n}\right|_{\partial \Omega}=0$. For any fixed $T>0$, if $G \in C\left([0, T] ; H^{3}(\Omega)\right)$, then $U \in C\left([0, T] ; H^{3}(\Omega)\right)$.

The next lemma will be used in the estimate of $\phi$ (cf. [6]).

Lemma 2.5. Let $\Omega \subset \mathbb{R}^{2}$ be any bounded domain with smooth boundary $\partial \Omega$. Then, for any function $H^{s}(\Omega) \ni f: \Omega \rightarrow \mathbb{R}$ satisfying $\left.\nabla f \cdot \mathbf{n}\right|_{\partial \Omega}=0$, it follows that

$$
\|f-\bar{f}\|_{H^{s}}^{2} \leq C\|\Delta f\|_{H^{s-2}}^{2},
$$

where $\bar{f}=\frac{1}{|\Omega|} \int_{\Omega} f d \mathbf{x}, C=C(s, \Omega)$ and the integer $s \geq 2$.

The global existence of weak solutions can be proved by using similar arguments in [6] and [32]. Otherwise, see the energy estimates given in the next section.

Lemma 2.6. Under the assumptions of Theorem 1.1, there exists a global weak solution $(\phi, \mu, U, \theta)$ to (1.1), (1.7) in the sense of distribution. 
3. Global regularity. In this section, we shall establish the regularity and uniqueness of the solution obtained in Lemma 2.6 under the assumptions of Theorem 1.1. The following theorem gives the key estimates.

TheOREm 3.1. Under the assumptions of Theorem 1.1, the solution obtained in Lemma 2.6 satisfies the following estimates:

$$
\begin{aligned}
& \|\phi\|_{C\left([0, T] ; H^{5}(\Omega)\right)}^{2}+\|\phi\|_{L^{2}\left([0, T] ; H^{7}(\Omega)\right)}^{2}+\|\mu\|_{C\left([0, T] ; H^{3}(\Omega)\right)}^{2}+\|\mu\|_{L^{2}\left([0, T] ; H^{5}(\Omega)\right)}^{2} \leq C, \\
& \|\theta\|_{C\left([0, T] ; H^{3}(\Omega)\right)}+\|\theta\|_{L^{2}\left([0, T] ; H^{4}(\Omega)\right)}+\|U\|_{C\left([0, T] ; H^{3}(\Omega)\right)} \leq C,
\end{aligned}
$$

for any $0<T<\infty$. Moreover, there exist constants $\gamma_{0}>0$ and $\eta_{0}(p) \geq 0$ which are independent of $t$ such that for any $t \geq 0$ and $p \in[2, \infty]$, it follows that

$$
\begin{gathered}
\|U(\cdot, t)\|^{2}+\|\phi(\cdot, t)\|_{H^{1}}^{2}+\|F(\phi(\cdot, t))\|_{L^{1}}+\int_{0}^{t}\|\nabla \mu(\cdot, \tau)\|^{2} d \tau \leq \gamma_{0}, \\
\|(\theta-\bar{\theta})(\cdot, t)\|_{L^{p}} \leq\left\|\theta_{0}-\bar{\theta}\right\|_{L^{p}} \exp \left\{-\eta_{0}(p) t\right\},
\end{gathered}
$$

where the constant $\eta_{0}(p)>0$ for $p \in[2, \infty)$ and $\eta_{0}(p)=0$ for $p=\infty$.

Due to the loss of viscosity and the appearance of nonhomogeneous terms in the velocity equation, our strategy for proving Theorem 3.1 is to first establish the full regularities of the nonhomogeneous terms using lower-order estimates of $U$. Then, by applying Lemma 2.4, we can build up the desired regularity of $U$. As indicated in the introduction, because of the lack of information of spatial derivatives of the solution on the boundary of the domain, our energy framework shall proceed towards lower-order spatial derivatives and temporal derivatives of the solution. Then by applying Lemma 2.2 we can obtain the estimates of higher-order spatial derivatives of the solution. The proof is based on several steps of careful energy estimates which are stated as a sequence of lemmas.

First, we observe that, due to the boundary conditions, upon integrating the first equation in (1.1) over $\Omega \times[0, t]$, it follows that

$$
\int_{\Omega} \phi(\mathbf{x}, t) d \mathbf{x}=\int_{\Omega} \phi_{0}(\mathbf{x}) d \mathbf{x} \equiv|\Omega| \bar{\phi}, \quad \forall t \geq 0 .
$$

Letting $\Phi=\phi-\bar{\phi}, \Theta=\theta-\bar{\theta}$ and plugging $\Phi$ and $\Theta$ into the original system (1.1) we have

$$
\left\{\begin{array}{l}
\Phi_{t}+U \cdot \nabla \Phi=\Delta \mu, \\
\mu=-\alpha \Delta \Phi+F^{\prime}(\phi), \\
U_{t}+U \cdot \nabla U+\nabla \tilde{P}=\mu \nabla \Phi+\Theta \mathbf{e}_{2}, \\
\Theta_{t}+U \cdot \nabla \Theta=\kappa \Delta \Theta, \\
\nabla \cdot U=0,
\end{array}\right.
$$

where $\tilde{P}=P-\bar{\theta} y$, and the initial and boundary conditions turn out to be

$$
\left\{\begin{array}{l}
(\Phi, \mu, U, \Theta)(\mathbf{x}, 0)=\left(\Phi_{0}, \mu_{0}, U_{0}, \Theta_{0}\right)(\mathbf{x}) \equiv\left(\phi_{0}-\bar{\phi}, \mu_{0}, U_{0}, \theta_{0}-\bar{\theta}\right)(\mathbf{x}), \\
\left.\nabla \Phi \cdot \mathbf{n}\right|_{\partial \Omega}=0,\left.\nabla \mu \cdot \mathbf{n}\right|_{\partial \Omega}=0,\left.U \cdot \mathbf{n}\right|_{\partial \Omega}=0,\left.\Theta\right|_{\partial \Omega}=0 .
\end{array}\right.
$$


In the rest of the paper, we shall work on the reformulated problem (3.1)-(3.2). Since $\bar{\phi}$ and $\bar{\theta}$ are constants, it suffices to prove the estimates stated in Theorem 3.1 for $\Phi$ and $\Theta$.

3.1. Uniform estimates of $(\Theta, U, \Phi, \mu)$. We first prove the $L^{2}$ version of the decay estimate of $\Theta$ together with some uniform estimate of the first-order derivative of $\Theta$. The result has been proved in 32 . However, for the sake of completeness and convenience of the readers, we still present the proof in the current paper. More importantly, we shall need some energy inequalities from the proof for later use.

Lemma 3.1. Under the assumptions of Theorem 1.1, there exists a constant $\beta_{0}$ independent of $t$ such that for any $t \geq 0$, it follows that

$$
\|\Theta(\cdot, t)\|^{2} \leq\left\|\Theta_{0}\right\|^{2} e^{-2 \beta_{0} t} \text { and } \int_{0}^{t}\|\nabla \Theta(\cdot, \tau)\|^{2} e^{\beta_{0} \tau} d \tau \leq \frac{1}{\kappa}\left\|\Theta_{0}\right\|^{2} .
$$

Proof. Taking the $L^{2}$ inner product of $(3.1)_{4}$ with $\Theta$ we have

$$
\frac{1}{2} \frac{d}{d t}\|\Theta\|^{2}+\kappa\|\nabla \Theta\|^{2}=0 .
$$

Since $\left.\Theta\right|_{\partial \Omega}=0$, Poincaré's inequality implies that

$$
\frac{d}{d t}\|\Theta\|^{2}+\frac{2 \kappa}{c_{0}}\|\Theta\|^{2} \leq 0,
$$

for some constant $c_{0}$ depending only on $\Omega$. (3.5) yields immediately that

$$
\|\Theta(\cdot, t)\|^{2} \leq\left\|\Theta_{0}\right\|^{2} e^{-2 \beta_{0} t},
$$

where $\beta_{0}=\kappa / c_{0}$. This proves the first part of (3.3).

Next, we multiply (3.4) by $e^{\beta_{0} t}$ to get

$$
\frac{d}{d t}\left(e^{\beta_{0} t}\|\Theta\|^{2}\right)+2 \kappa e^{\beta_{0} t}\|\nabla \Theta\|^{2}=\beta_{0} e^{\beta_{0} t}\|\Theta\|^{2} .
$$

Then (3.6) implies that

$$
\frac{d}{d t}\left(e^{\beta_{0} t}\|\Theta\|^{2}\right)+2 \kappa e^{\beta_{0} t}\|\nabla \Theta\|^{2} \leq \beta_{0} e^{-\beta_{0} t}\left\|\Theta_{0}\right\|^{2} .
$$

For any $t>0$, upon integrating in time we obtain

$$
e^{\beta_{0} t}\|\Theta(\cdot, t)\|^{2}-\left\|\Theta_{0}\right\|^{2}+2 \kappa \int_{0}^{t} e^{\beta_{0} \tau}\|\nabla \Theta(\cdot, \tau)\|^{2} d \tau \leq\left(1-e^{-\beta_{0} t}\right)\left\|\Theta_{0}\right\|^{2},
$$

which implies the second part of (3.3) immediately. This completes the proof.

Next, with the help of the above lemma, we prove the uniform estimates of $U, \Phi$ and $\mu$ stated in Theorem 3.1.

Lemma 3.2. Under the assumptions of Theorem 1.1, there exists a constant $\gamma_{1}$ independent of $t$ such that for any $t \geq 0$, it follows that

$$
\|U(\cdot, t)\|^{2}+\|\Phi(\cdot, t)\|_{H^{1}}^{2}+\|F(\phi(\cdot, t))\|_{L^{1}}+\int_{0}^{t}\|\nabla \mu(\cdot, \tau)\|^{2} d \tau \leq \gamma_{1} .
$$


Proof. Taking the $L^{2}$ inner product of $(3.1)_{3}$ with $U$ we have

$$
\frac{1}{2} \frac{d}{d t}\|U\|^{2}=\int_{\Omega} \mu(\nabla \Phi \cdot U) d \mathbf{x}+\int_{\Omega} \Theta \mathbf{e}_{2} \cdot U d \mathbf{x}
$$

Taking the $L^{2}$ inner product of $(3.1)_{1}$ with $\mu$ we have

$$
\frac{d}{d t}\left(\frac{\alpha}{2}\|\nabla \Phi\|^{2}+\int_{\Omega} F(\phi) d \mathbf{x}\right)+\|\nabla \mu\|^{2}=-\int_{\Omega} \mu(\nabla \Phi \cdot U) d \mathbf{x} .
$$

Adding (3.8) and (3.9) we get

$$
\frac{d}{d t}\left(\frac{1}{2}\|U\|^{2}+\frac{\alpha}{2}\|\nabla \Phi\|^{2}+\int_{\Omega} F(\phi) d \mathbf{x}\right)+\|\nabla \mu\|^{2}=\int_{\Omega} \Theta \mathbf{e}_{2} \cdot U d \mathbf{x}
$$

Applying the Cauchy-Schwarz inequality to the right-hand side (RHS) of (3.10) we obtain

$$
\frac{d}{d t}\left(\frac{1}{2}\|U\|^{2}+\frac{\alpha}{2}\|\nabla \Phi\|^{2}+\int_{\Omega} F(\phi) d \mathbf{x}\right)+\|\nabla \mu\|^{2} \leq e^{-\beta_{0} t}\|U\|^{2}+e^{\beta_{0} t}\|\Theta\|^{2},
$$

which implies, by (3.6), that

$$
\frac{d}{d t}\left(\frac{1}{2}\|U\|^{2}+\frac{\alpha}{2}\|\nabla \Phi\|^{2}+\int_{\Omega} F(\phi) d \mathbf{x}\right)+\|\nabla \mu\|^{2} \leq e^{-\beta_{0} t}\|U\|^{2}+e^{-\beta_{0} t}\left\|\Theta_{0}\right\|^{2} .
$$

By dropping $\|\nabla \mu\|^{2}$ from the left-hand side (LHS) of (3.11) we get

$$
\frac{d}{d t}\left(\frac{1}{2}\|U\|^{2}+\frac{\alpha}{2}\|\nabla \Phi\|^{2}+\int_{\Omega} F(\phi) d \mathbf{x}\right) \leq e^{-\beta_{0} t}\|U\|^{2}+e^{-\beta_{0} t}\left\|\Theta_{0}\right\|^{2} .
$$

Since $F \geq 0$, we have

$$
\frac{d}{d t}(E(t)) \leq 2 e^{-\beta_{0} t} E(t)+e^{-\beta_{0} t}\left\|\Theta_{0}\right\|^{2},
$$

where

$$
E(t) \equiv \frac{1}{2}\|U\|^{2}+\frac{\alpha}{2}\|\nabla \Phi\|^{2}+\int_{\Omega} F(\phi) d \mathbf{x} .
$$

Gronwall's inequality then gives

$$
E(t) \leq e^{2 / \beta_{0}}\left(E(0)+\frac{1}{\beta_{0}}\left(1-e^{-\beta_{0} t}\right)\left\|\Theta_{0}\right\|^{2}\right),
$$

which implies that

$$
\frac{1}{2}\|U\|^{2}+\frac{\alpha}{2}\|\nabla \Phi\|^{2}+\int_{\Omega} F(\phi) d \mathbf{x} \leq e^{2 / \beta_{0}}\left(E(0)+\frac{1}{\beta_{0}}\left\|\Theta_{0}\right\|^{2}\right), \quad \forall t \geq 0 .
$$

Plugging (3.12) into (3.11) we have

$$
\frac{d}{d t}(E(t))+\|\nabla \mu\|^{2} \leq e^{-\beta_{0} t}\left(e^{2 / \beta_{0}}\left(E(0)+\frac{1}{\beta_{0}}\left\|\Theta_{0}\right\|^{2}\right)+\left\|\Theta_{0}\right\|^{2}\right) .
$$

Upon integrating the above inequality in time we obtain

$$
E(t)+\int_{0}^{t}\|\nabla \mu(\cdot, \tau)\|^{2} d \tau \leq \frac{1}{\beta_{0}}\left(e^{2 / \beta_{0}}\left(E(0)+\frac{1}{\beta_{0}}\left\|\Theta_{0}\right\|^{2}\right)+\left\|\Theta_{0}\right\|^{2}\right)+E(0) \equiv \alpha_{0} .
$$

By definition we have

$$
\frac{1}{2}\|U\|^{2}+\frac{\alpha}{2}\|\nabla \Phi\|^{2}+\int_{\Omega} F(\phi) d \mathbf{x}+\int_{0}^{t}\|\nabla \mu(\cdot, \tau)\|^{2} d \tau \leq \alpha_{0} .
$$


Since $\bar{\phi}$ is the average of $\phi$ over $\Omega$, applying Poincaré's inequality we have

$$
\|\Phi\|^{2}=\|\phi-\bar{\phi}\|^{2} \leq c_{0}\|\nabla \Phi\|^{2},
$$

which, together with (3.13), proves (3.7). This completes the proof.

3.2. $H^{2}$ estimate of $\mu, H^{4}$ estimate of $\Phi$ and $H^{1}$ estimate of $U$. We now proceed towards higher-order estimates of the solution. Fix any $0<T<\infty$. In the rest of this section, the time is restricted to be within the interval $[0, T]$ until specified otherwise. The constants appearing in the energy estimates may depend on $T$ in general, but are finite for any $0 \leq T<\infty$. First, with the help of Lemma 3.2, we prove the $H^{3}$ estimate of $\Phi$.

Lemma 3.3. Under the assumptions of Theorem 1.1, it follows that

$$
\int_{0}^{T}\|\Phi(\cdot, t)\|_{H^{3}}^{2} d t \leq C .
$$

Proof. Taking the $L^{2}$ inner product of $(3.1)_{1}$ with $\Phi$, after integrating by parts we have

$$
\frac{1}{2} \frac{d}{d t}\|\Phi\|^{2}=\int_{\Omega} \Phi \Delta \mu \mathbf{x}=-\int_{\Omega} \nabla \mu \cdot \nabla \Phi d \mathbf{x}=-\alpha\|\Delta \Phi\|^{2}-\int_{\Omega} F^{\prime \prime}(\phi)|\nabla \Phi|^{2} d \mathbf{x},
$$

which gives

$$
\frac{d}{d t}\|\Phi\|^{2}+2 \alpha\|\Delta \Phi\|^{2} \leq 2 F_{3}\|\nabla \Phi\|^{2},
$$

where we have used the condition $\left(H_{3}\right)$. Integrating (3.15) over time and using (3.7) we have

$$
\|\Phi(\cdot, t)\|^{2}+2 \alpha \int_{0}^{t}\|\Delta \Phi\|^{2} d \tau \leq 2 t F_{3} \gamma_{1}+\left\|\Phi_{0}\right\|^{2} \leq 2 T F_{3} \gamma_{1}+\left\|\Phi_{0}\right\|^{2}, \quad \forall t \in[0, T],
$$

which implies that

$$
\sup _{t \in[0, T]}\|\Phi(\cdot, t)\|^{2}+2 \alpha \int_{0}^{T}\|\Delta \Phi\|^{2} d t \leq 2 T F_{3} \gamma_{0}+\left\|\Phi_{0}\right\|^{2} .
$$

By Lemma 2.5 we then have

$$
\sup _{t \in[0, T]}\|\Phi(\cdot, t)\|^{2}+\int_{0}^{T}\|\Phi\|_{H^{2}}^{2} d t \leq C .
$$

To estimate $\|\Phi\|_{H^{3}}^{2}$, by Lemma $2.5,\left(H_{2}\right)$, Lemma 2.1 and (3.7) we have

$$
\begin{aligned}
\|\Phi\|_{H^{3}}^{2} & \leq C\|\Delta \Phi\|_{H^{1}}^{2} \\
& \leq C\left(\|\Delta \Phi\|^{2}+\|\nabla \mu\|^{2}+\left\|F^{\prime \prime}(\phi) \nabla \Phi\right\|^{2}\right) \\
& \leq C\left(\|\Delta \Phi\|^{2}+\|\nabla \mu\|^{2}+\|\phi\|_{L^{4(p-2)}}^{2(p-2)}\|\nabla \Phi\|_{L^{4}}^{2}+\|\nabla \Phi\|^{2}\right) \\
& \leq C\left(\|\Delta \Phi\|^{2}+\|\nabla \mu\|^{2}+\left(\|\Phi\|_{H^{1}}^{2(p-2)}+\|\bar{\phi}\|_{H^{1}}^{2(p-2)}\right)\|\nabla \Phi\|_{H^{1}}^{2}+\|\nabla \Phi\|^{2}\right) \\
& \leq C\left(\|\Phi\|_{H^{2}}^{2}+\|\nabla \mu\|^{2}\right) .
\end{aligned}
$$


From (3.7) and (3.16) we see that the RHS of (3.17) is integrable in time over $[0, T]$, which yields

$$
\int_{0}^{T}\|\Phi(\cdot, t)\|_{H^{3}}^{2} d t \leq C
$$

This completes the proof.

Second, we improve the regularity of $U$.

LEMmA 3.4. Under the assumptions of Theorem 1.1, it follows that

$$
\sup _{t \in[0, T]}\|U(\cdot, t)\|_{H^{1}}^{2} \leq C .
$$

Proof. By virtue of the boundary condition, the incompressibility condition and Lemma 2.3 with $s=1, p=2$ we have

$$
\|U\|_{H^{1}}^{2} \leq C\left(\|\omega\|^{2}+\|U\|^{2}\right)
$$

where $\omega=v_{x}-u_{y}$ is the $2 \mathrm{D}$ vorticity. In view of Lemma 3.2, we see that it suffices to estimate $\|\omega\|^{2}$ in order to show (3.18). For this purpose, taking the curl of the velocity equation we get

$$
\omega_{t}+U \cdot \nabla \omega=\mu_{x} \Phi_{y}-\mu_{y} \Phi_{x}+\Theta_{x} .
$$

Taking the $L^{2}$ inner product of (3.19) with $\omega$ and applying Hölder's inequality we have

$$
\frac{1}{2} \frac{d}{d t}\|\omega\|^{2} \leq\left(\left\|\mu_{x} \Phi_{y}-\mu_{y} \Phi_{x}\right\|+\left\|\Theta_{x}\right\|\right)\|\omega\|,
$$

which implies that

$$
\frac{d}{d t}\|\omega\| \leq 2\|\nabla \mu\|\|\nabla \Phi\|_{L^{\infty}}+\|\nabla \Theta\| .
$$

Upon integrating in time using Hölder's inequality and Lemma 2.1 (i) we have

$$
\begin{aligned}
\sup _{t \in[0, T]}\|\omega(\cdot, t)\| \leq & \int_{0}^{T}\left(2\|\nabla \mu\|\|\nabla \Phi\|_{L^{\infty}}+\|\nabla \Theta\|\right) d t+\left\|\omega_{0}\right\| \\
\leq & C\left(\int_{0}^{T}\|\nabla \mu\|^{2} d t\right)^{\frac{1}{2}}\left(\int_{0}^{T}\|\Phi\|_{H^{3}}^{2} d t\right)^{\frac{1}{2}} \\
& +\left(\int_{0}^{T} e^{\beta_{0} t / 2}\|\nabla \Theta\|^{2} d t\right)^{\frac{1}{2}}\left(\int_{0}^{T} e^{-\beta_{0} t / 2} d t\right)^{\frac{1}{2}}+\left\|\omega_{0}\right\| .
\end{aligned}
$$

From Lemma 3.2 and Lemma 3.3 we see that the first term on the RHS of (3.20) is bounded, and the second term is also bounded according to Lemma 3.1. Therefore, we have $\sup _{t \in[0, T]}\|\omega(\cdot, t)\|^{2} \leq C$. This completes the proof.

As indicated in the introduction, due to the lack of information of spatial derivatives of the solution on the boundary of the domain, we need to estimate temporal derivatives of the solution in order to improve spatial regularity. The next lemma gives the first estimate of the temporal derivative of the solution.

LEMma 3.5. Under the assumptions of Theorem 1.1, it follows that

$$
\sup _{t \in[0, T]}\|\Phi(\cdot, t)\|_{H^{2}}^{2}+\int_{0}^{T}\left\|\Phi_{t}\right\|^{2} d t \leq C .
$$


Proof. Taking the $L^{2}$ inner product of $(3.1)_{1}$ with $\Phi_{t}$ we have

$$
\left\|\Phi_{t}\right\|^{2}+\int_{\Omega} \Phi_{t}(U \cdot \nabla \Phi) d \mathbf{x}=\int_{\Omega} \Phi_{t} \Delta \mu d \mathbf{x} .
$$

Using the boundary condition we calculate the RHS of (3.22) as follows:

$$
\begin{aligned}
\int_{\Omega} \Phi_{t} \Delta \mu d \mathbf{x} & =\int_{\Omega} \mu \Delta \Phi_{t} d \mathbf{x} \\
& =-\frac{d}{d t}\left(\frac{\alpha}{2}\|\Delta \Phi\|^{2}\right)+\int_{\Omega} F^{\prime}(\phi) \Delta \Phi_{t} d \mathbf{x} \\
& =-\frac{d}{d t}\left(\frac{\alpha}{2}\|\Delta \Phi\|^{2}+\frac{1}{2} \int_{\Omega} F^{\prime \prime}(\phi)|\nabla \Phi|^{2} d \mathbf{x}\right)+\frac{1}{2} \int_{\Omega} F^{\prime \prime \prime}(\phi) \Phi_{t}|\nabla \Phi|^{2} d \mathbf{x} .
\end{aligned}
$$

Plugging (3.23) into (3.22) we get

$$
\begin{aligned}
& \frac{d}{d t}\left(\frac{\alpha}{2}\|\Delta \Phi\|^{2}+\frac{1}{2} \int_{\Omega} F^{\prime \prime}(\phi)|\nabla \Phi|^{2} d \mathbf{x}\right)+\left\|\Phi_{t}\right\|^{2} \\
= & \frac{1}{2} \int_{\Omega} F^{\prime \prime \prime}(\phi) \Phi_{t}|\nabla \Phi|^{2} d \mathbf{x}-\int_{\Omega} \Phi_{t}(U \cdot \nabla \Phi) d \mathbf{x} .
\end{aligned}
$$

Using the Cauchy-Schwarz inequality, $\left(H_{2}\right)$ and Lemma 3.2 we estimate the first term on the RHS of (3.24) as follows:

$$
\begin{aligned}
\left.\left|\frac{1}{2} \int_{\Omega} F^{\prime \prime \prime}(\phi) \Phi_{t}\right| \nabla \Phi\right|^{2} d \mathbf{x} \mid & \leq \frac{1}{4}\left\|\Phi_{t}\right\|^{2}+\frac{1}{4} \int_{\Omega}\left|F^{\prime \prime \prime}(\phi)\right|^{2}|\nabla \Phi|^{4} d \mathbf{x} \\
& \leq \frac{1}{4}\left\|\Phi_{t}\right\|^{2}+C \int_{\Omega}\left(|\phi|^{2(p-3)}+1\right)|\nabla \Phi|^{4} d \mathbf{x} \\
& \leq \frac{1}{4}\left\|\Phi_{t}\right\|^{2}+C\|\phi\|_{L^{4(p-3)}}^{2(p-3)}\|\nabla \Phi\|_{L^{8}}^{4}+C\|\nabla \Phi\|_{L^{4}}^{4} \\
& \leq \frac{1}{4}\left\|\Phi_{t}\right\|^{2}+C\|\phi\|_{H^{1}}^{2(p-3)}\|\nabla \Phi\|_{L^{8}}^{4}+C\|\nabla \Phi\|_{L^{4}}^{4} \\
& \leq \frac{1}{4}\left\|\Phi_{t}\right\|^{2}+C\left(\|\nabla \Phi\|_{L^{8}}^{4}+\|\nabla \Phi\|_{L^{4}}^{4}\right) .
\end{aligned}
$$

Lemma 2.1 (iii)-(v) and Lemma 3.2 then give

$$
\begin{aligned}
\|\nabla \Phi\|_{L^{4}}^{4}+\|\nabla \Phi\|_{L^{8}}^{4} & \leq C\left(\|\nabla \Phi\|^{2}\left\|\nabla^{2} \Phi\right\|^{2}+\|\nabla \Phi\|^{4}+\|\nabla \Phi\|^{2}\left\|\nabla^{2} \Phi\right\|_{L^{4}}^{2}+\|\nabla \Phi\|^{4}\right) \\
& \leq C\|\nabla \Phi\|^{2}\left(\left\|\nabla^{2} \Phi\right\|^{2}+\|\nabla \Phi\|^{2}+\left\|\nabla^{2} \Phi\right\|_{H^{1}}^{2}\right) \\
& \leq C\|\Phi\|_{H^{3}}^{2}
\end{aligned}
$$

So we update $(3.25)$ as

$$
\left.\left|\frac{1}{2} \int_{\Omega} F^{\prime \prime \prime}(\phi) \Phi_{t}\right| \nabla \Phi\right|^{2} d \mathbf{x} \mid \leq \frac{1}{4}\left\|\Phi_{t}\right\|^{2}+C\|\Phi\|_{H^{3}}^{2} .
$$


The second term on the RHS of (3.24) is estimated as

$$
\begin{aligned}
\left|-\int_{\Omega} \Phi_{t}(U \cdot \nabla \Phi) d \mathbf{x}\right| & \leq \frac{1}{4}\left\|\Phi_{t}\right\|^{2}+\|U \cdot \nabla \Phi\|^{2} \\
& \leq \frac{1}{4}\left\|\Phi_{t}\right\|^{2}+\|U\|_{L^{4}}^{2}\|\nabla \Phi\|_{L^{4}}^{2} \\
& \leq \frac{1}{4}\left\|\Phi_{t}\right\|^{2}+C\|U\|_{H^{1}}^{2}\|\nabla \Phi\|_{H^{1}}^{2} \\
& \leq \frac{1}{4}\left\|\Phi_{t}\right\|^{2}+C\|\Phi\|_{H^{2}}^{2},
\end{aligned}
$$

where we used Lemma 3.4 for $\|U\|_{H^{1}}^{2}$. Plugging (3.26) and (3.27) into (3.24) we have

$$
\frac{d}{d t}\left(\frac{\alpha}{2}\|\Delta \Phi\|^{2}+\frac{1}{2} \int_{\Omega} F^{\prime \prime}(\phi)|\nabla \Phi|^{2} d \mathbf{x}\right)+\frac{1}{2}\left\|\Phi_{t}\right\|^{2} \leq C\|\Phi\|_{H^{3}}^{2} .
$$

Integrating (3.28) over time and using Lemma 3.3 we find

$$
\sup _{t \in[0, T]}\left(\alpha\|\Delta \Phi\|^{2}+\int_{\Omega} F^{\prime \prime}(\phi)|\nabla \Phi|^{2} d \mathbf{x}\right)+\int_{0}^{T}\left\|\Phi_{t}\right\|^{2} d t \leq C,
$$

which implies, by $\left(H_{3}\right)$, that

$$
\sup _{t \in[0, T]} \alpha\|\Delta \Phi\|^{2}+\int_{0}^{T}\left\|\Phi_{t}\right\|^{2} d t \leq C+F_{3} \sup _{t \in[0, T]}\|\nabla \Phi\|^{2} \leq C,
$$

where we used (3.7). We complete the proof by applying Lemma 2.5 to the above estimate.

As by-products of previous lemmas, we have

LEMmA 3.6. Under the assumptions of Theorem 1.1, it follows that

$$
\sup _{t \in[0, T]}\|\mu(\cdot, t)\|^{2}+\int_{0}^{T}\left(\|\mu(\cdot, t)\|_{H^{2}}^{2}+\|\Phi(\cdot, t)\|_{H^{4}}^{2}\right) d t \leq C .
$$

Proof. First, we note, by (3.21) and Lemma 2.1 (i), that it follows that

$$
\sup _{t \in[0, T]}\|\Phi\|_{L^{\infty}} \leq C
$$

which implies, according to $\left(H_{2}\right)$, that

$$
\sup _{t \in[0, T]}\left\|F^{(p-n)}(\phi)\right\|_{L^{\infty}} \leq F_{1} C(p, n)\left(\|\Phi\|_{L^{\infty}}^{(p-n)}+\|\bar{\phi}\|_{L^{\infty}}^{(p-n)}\right)+F_{2} \leq C, \quad n=1, \ldots, 6 .
$$

From $(3.1)_{2},(3.21)$ and $(3.30)$ we then have

$$
\begin{aligned}
\sup _{t \in[0, T]}\|\mu(\cdot, t)\|^{2} & \leq C \sup _{t \in[0, T]}\left(\|\Delta \Phi\|^{2}+\left\|F^{\prime}(\phi)\right\|^{2}\right) \\
& \leq C \sup _{t \in[0, T]}\left(\|\Delta \Phi\|^{2}+\left\|F^{\prime}(\phi)\right\|_{L^{\infty}}^{2}|\Omega|\right) \\
& \leq C .
\end{aligned}
$$

Second, since $\left.\nabla \mu \cdot \mathbf{n}\right|_{\partial \Omega}=0$, we get from Lemma 2.3 that

$$
\|\nabla \mu\|_{H^{1}}^{2} \leq C\left(\|\Delta \mu\|^{2}+\|\nabla \mu\|^{2}\right) .
$$


By $(3.1)_{1}$ and Lemmas $3.4-3.5$ we have

$$
\begin{aligned}
\|\Delta \mu\|^{2} & \leq C\left(\left\|\Phi_{t}\right\|^{2}+\|U \cdot \nabla \Phi\|^{2}\right) \\
& \leq C\left(\left\|\Phi_{t}\right\|^{2}+\|U\|_{H^{1}}^{2}\|\nabla \Phi\|_{H^{1}}^{2}\right) \\
& \leq C\left(\left\|\Phi_{t}\right\|^{2}+1\right),
\end{aligned}
$$

which implies that

$$
\|\nabla \mu\|_{H^{1}}^{2} \leq C\left(\left\|\Phi_{t}\right\|^{2}+1+\|\nabla \mu\|^{2}\right) .
$$

From (3.7) and (3.21) we know that the RHS of (3.32) is integrable in time over $[0, T]$. We then have

$$
\int_{0}^{T}\|\nabla \mu\|_{H^{1}}^{2} d t \leq C
$$

which, together with (3.31), implies that

$$
\int_{0}^{T}\|\mu\|_{H^{2}}^{2} d t \leq C
$$

For the $H^{4}$ estimate of $\Phi$, we have, by Lemma 2.5:

$$
\|\Phi\|_{H^{4}}^{2} \leq C\|\Delta \Phi\|_{H^{2}}^{2} \leq C\left(\|\mu\|_{H^{2}}^{2}+\left\|F^{\prime}(\phi)\right\|_{H^{2}}^{2}\right) .
$$

By $\left(H_{2}\right),(3.30)$ and $(3.21)$ we have

$$
\begin{aligned}
\left\|F^{\prime}(\phi)\right\|_{H^{2}}^{2} & \leq C\left(\left\|F^{\prime}(\phi)\right\|_{L^{\infty}}^{2}|\Omega|+\left\|F^{\prime \prime}(\phi)\right\|_{L^{\infty}}^{2}\left(\|\nabla \Phi\|^{2}+\left\|\nabla^{2} \Phi\right\|^{2}\right)+\left\|F^{\prime \prime \prime}(\phi)\right\|_{L^{\infty}}^{2}\|\nabla \Phi\|_{L^{4}}^{4}\right) \\
& \leq C\left(1+\|\Phi\|_{H^{2}}^{2}+\|\Phi\|_{H^{2}}^{4}\right) \leq C,
\end{aligned}
$$

which, together with (3.33)-(3.34), implies that

$$
\int_{0}^{T}\|\Phi\|_{H^{4}}^{2} d t \leq C
$$

This completes the proof.

3.3. $H^{2}$ estimates of $\Theta$ and $W^{1, p}$ estimate of $U$. Due to nonlinear convection, in order to gain more regularities on $\Phi$, we shall need the estimates of $\|U\|_{C\left([0, T] ; W^{1, p}(\Omega)\right)}(2<$ $p<\infty)$ and $\left\|U_{t}\right\|_{L^{2}\left([0, T] ; L^{2}(\Omega)\right)}$. Due to the structure of the vorticity equation (3.19) and the Sobolev embedding $H^{2} \hookrightarrow W^{1, p}$ for $2<p<\infty$, we need to estimate $\|\Theta\|_{H^{2}}$ first. The desired estimate is given in the next lemma.

LEMmA 3.7. Under the assumptions of Theorem 1.1, it follows that

$$
\sup _{t \in[0, T]}\|\Theta(\cdot, t)\|_{H^{1}}^{2}+\int_{0}^{T}\|\Theta(\cdot, t)\|_{H^{2}}^{2} d t \leq C .
$$

Proof. Taking the $L^{2}$ inner product of $(3.1)_{4}$ with $\Theta_{t}$ we find

$$
\frac{\kappa}{2} \frac{d}{d t}\|\nabla \Theta\|^{2}+\left\|\Theta_{t}\right\|^{2}=-\int_{\Omega}(U \cdot \nabla \Theta) \Theta_{t} d \mathbf{x} .
$$

We estimate the RHS of (3.36) as follows: First, using the Cauchy-Schwarz inequality, we get

$$
\left|-\int_{\Omega}(U \cdot \nabla \Theta) \Theta_{t} d \mathbf{x}\right| \leq\|U \cdot \nabla \Theta\|^{2}+\frac{1}{4}\left\|\Theta_{t}\right\|^{2} .
$$


Using Lemma 2.1 and (3.18) we have

$$
\begin{aligned}
\|U \cdot \nabla \Theta\|^{2} & \leq\|U\|_{L^{4}}^{2}\|\nabla \Theta\|_{L^{4}}^{2} \\
& \leq C\|U\|_{H^{1}}^{2}\|\nabla \Theta\|_{L^{4}}^{2} \\
& \leq C\|\nabla \Theta\|_{L^{4}}^{2} .
\end{aligned}
$$

So we update (3.36) as

$$
\frac{\kappa}{2} \frac{d}{d t}\|\nabla \Theta\|^{2}+\frac{3}{4}\left\|\Theta_{t}\right\|^{2} \leq \bar{C}\|\nabla \Theta\|_{L^{4}}^{2}
$$

where we used the overline to specify the constant in order to avoid confusion. For the RHS of (3.37), applying Lemma 2.1 (iv) to $\nabla \Theta$ we get

$$
\begin{aligned}
\bar{C}\|\nabla \Theta\|_{L^{4}}^{2} & \leq C\left(\|\nabla \Theta\|\left\|D^{2} \Theta\right\|+\|\nabla \Theta\|^{2}\right) \\
& \leq C(\delta)\|\nabla \Theta\|^{2}+\delta\left\|D^{2} \Theta\right\|^{2},
\end{aligned}
$$

where $\delta$ is a number to be determined. Since $\left.\Theta\right|_{\partial \Omega}=0$, using (3.1) 4 and Lemma 2.2 with $m=0$ and $p=2$ we have

$$
\|\Theta\|_{H^{2}}^{2} \leq C\left(\left\|\Theta_{t}\right\|^{2}+\|U \cdot \nabla \Theta\|^{2}\right) .
$$

For the second term on the RHS of (3.39), we use (3.38) to get

$$
\|U \cdot \nabla \Theta\|^{2} \leq c\|U\|_{H^{1}}^{2}\|\nabla \Theta\|_{L^{4}}^{2} \leq C\left(\|\nabla \Theta\|\left\|D^{2} \Theta\right\|+\|\nabla \Theta\|^{2}\right) .
$$

Then, using the Cauchy-Schwarz inequality, we update (3.39) as

$$
\begin{aligned}
\|\Theta\|_{H^{2}}^{2} & \leq C\left(\left\|\Theta_{t}\right\|^{2}+\|\nabla \Theta\|\left\|D^{2} \Theta\right\|+\|\nabla \Theta\|^{2}\right) \\
& \leq C\left(\left\|\Theta_{t}\right\|^{2}+\|\nabla \Theta\|^{2}\right)+\frac{1}{2}\|\Theta\|_{H^{2}}^{2}
\end{aligned}
$$

which implies that

$$
\|\Theta\|_{H^{2}}^{2} \leq \widehat{C}\left(\left\|\Theta_{t}\right\|^{2}+\|\nabla \Theta\|^{2}\right)
$$

By choosing $\delta=1 /(4 \widehat{C})$ in (3.38), and coupling the result with (3.40) we have

$$
\bar{C}\|\nabla \Theta\|_{L^{4}}^{2} \leq C\|\nabla \Theta\|^{2}+\frac{1}{4}\left\|\Theta_{t}\right\|^{2}
$$

Combining (3.37) with (3.41) we get

$$
\frac{\kappa}{2} \frac{d}{d t}\|\nabla \Theta\|^{2}+\frac{1}{2}\left\|\Theta_{t}\right\|^{2} \leq C\|\nabla \Theta\|^{2} .
$$

Upon integrating (3.42) in time and using Lemma 3.1 we have

$$
\sup _{t \in[0, T]}\|\nabla \Theta(\cdot, t)\|^{2}+\int_{0}^{T}\left\|\Theta_{t}\right\|^{2} d t \leq C .
$$

Since $\left.\Theta\right|_{\partial \Omega}=0$, Poincaré's inequality gives

$$
\sup _{t \in[0, T]}\|\Theta(\cdot, t)\|_{H^{1}}^{2}+\int_{0}^{T}\left\|\Theta_{t}\right\|^{2} d t \leq C,
$$

which, together with (3.40), implies (3.35). This completes the proof.

Using Lemma 3.7 we now establish the estimates of $\|U\|_{C\left([0, T] ; W^{1, p}(\Omega)\right)}(2<p<\infty)$ and $\left\|U_{t}\right\|_{L^{2}\left([0, T] ; L^{2}(\Omega)\right)}$. 
LEMma 3.8. Under the assumptions of Theorem 1.1, it follows that

$$
\sup _{t \in[0, T]}\|U(\cdot, t)\|_{W^{1, p}}^{2} \leq C(p), \quad \forall 2<p<\infty, \quad \text { and } \int_{0}^{T}\left\|U_{t}(\cdot, t)\right\|^{2} d t \leq C .
$$

Proof. By virtue of Lemma 2.3 and Lemma 3.4, it suffices to estimate $\|\omega\|_{L^{p}}^{2}$ in order to obtain $\|U\|_{W^{1, p}}^{2}$. For this purpose, for any $2<p<\infty$, taking the $L^{2}$ inner product of the vorticity equation (3.19) with $|\omega|^{p-2} \omega$ we have

$$
\frac{1}{p} \frac{d}{d t}\|\omega\|_{L^{p}}^{p} \leq\left(2\|\nabla \mu\|_{L^{2 p}}\|\nabla \Phi\|_{L^{2 p}}+\|\nabla \Theta\|_{L^{p}}\right)\|\omega\|_{L^{p}}^{p-1}
$$

which, together with Lemma 2.1 (iii), gives

$$
\begin{aligned}
\frac{d}{d t}\|\omega\|_{L^{p}} & \leq 2\|\nabla \mu\|_{L^{2 p}}\|\nabla \Phi\|_{L^{2 p}}+\|\nabla \Theta\|_{L^{p}} \\
& \leq C(p)\left(\|\nabla \mu\|_{H^{1}}\|\Phi\|_{H^{2}}+\|\Theta\|_{H^{2}}\right) .
\end{aligned}
$$

Upon integrating (3.45) in time and using Hölder's inequality we have

$$
\|\omega\|_{L^{p}} \leq C(p)\left[\left(\int_{0}^{T}\|\nabla \mu\|_{H^{1}}^{2} d\right)^{\frac{1}{2}}\left(\int_{0}^{T}\|\Phi\|_{H^{2}}^{2} d\right)^{\frac{1}{2}}+T^{\frac{1}{2}}\left(\int_{0}^{T}\|\Theta\|_{H^{2}}^{2} d t\right)^{\frac{1}{2}}\right]+\left\|\omega_{0}\right\|_{L^{p}} .
$$

From (3.29) and (3.39) we see that the temporal integrals on the RHS of the above estimate are bounded. Then we have

$$
\sup _{t \in[0, T]}\|\omega(\cdot, t)\|_{L^{p}} \leq C(p), \quad \forall 2<p<\infty,
$$

which gives

$$
\sup _{t \in[0, T]}\|U(\cdot, t)\|_{W^{1, p}} \leq C(p), \quad \forall 2<p<\infty .
$$

By (3.46) with $p=3$ and Lemma 2.1 (iii) we then have

$$
\|U\|_{L^{\infty}} \leq C\|U\|_{W^{1,3}} \leq C .
$$

To estimate $\left\|U_{t}\right\|^{2}$, taking the $L^{2}$ inner product of $(3.1)_{3}$ with $U_{t}$ we have

$$
\begin{aligned}
\left\|U_{t}\right\|^{2} & \leq\|U \cdot \nabla U\|^{2}+\|\mu \nabla \Phi\|^{2}+\|\Theta\|^{2} \\
& \leq\|U\|_{L^{\infty}}^{2}\|\nabla U\|^{2}+\|\mu\|_{H^{1}}^{2}\|\Phi\|_{H^{2}}^{2}+\left\|\Theta_{0}\right\|^{2} \\
& \leq C\left(\|\mu\|^{2}+\|\nabla \mu\|^{2}+1\right) \\
& \leq C\left(\|\nabla \mu\|^{2}+1\right),
\end{aligned}
$$

where we have used Lemma 3.1 for $\|\Theta\|^{2}$, (3.46) for $\|\nabla U\|^{2}$, (3.47) for $\|U\|_{L^{\infty}}^{2}$, Lemma 3.5 for $\|\Phi\|_{H^{2}}^{2}$ and Lemma 3.6 for $\|\mu\|^{2}$. Therefore, the lemma follows from (3.48) and Lemma 3.2. This completes the proof.

3.4. $H^{3}$ estimates of $\mu$ and $H^{5}$ estimate of $\Phi$. The next lemma serves as one of the building blocks for building up the full regularity of $U$. Indeed, the lemma gives the $H^{3}$ estimate of $\mu \nabla \phi$.

LEMma 3.9. Under the assumptions of Theorem 1.1, it follows that

$$
\sup _{t \in[0, T]}\left(\|\mu(\cdot, t)\|_{H^{3}}^{2}+\|\Phi(\cdot, t)\|_{H^{5}}^{2}\right) \leq C .
$$


Proof. The lemma is proved by estimating temporal derivatives of the solution. Taking the $L^{2}$ inner product of $(3.1)_{1}$ with $\mu_{t}$ we have

$$
\frac{1}{2} \frac{d}{d t}\|\nabla \mu\|^{2}+\alpha\left\|\nabla \Phi_{t}\right\|^{2}=-\int_{\Omega}\left[F^{\prime \prime}(\phi) \Phi_{t}^{2}+\mu_{t}(U \cdot \nabla \Phi)\right] d \mathbf{x} .
$$

Using (3.30), (3.47) and (3.21) we estimate the RHS of (3.50) as follows:

$$
\begin{aligned}
& \left|\int_{\Omega}\left[F^{\prime \prime}(\phi) \Phi_{t}^{2}+\mu_{t}(U \cdot \nabla \Phi)\right] d \mathbf{x}\right| \\
& \quad \leq\left\|F^{\prime \prime}(\phi)\right\|_{L^{\infty}}\left\|\Phi_{t}\right\|^{2}+C\|U \cdot \nabla \Phi\|^{2}+\frac{1}{8 \alpha}\left\|\mu_{t}\right\|^{2} \\
& \quad \leq C\left\|\Phi_{t}\right\|^{2}+C\|U\|_{L^{\infty}}^{2}\|\nabla \Phi\|^{2}+\frac{1}{4 \alpha}\left(\alpha^{2}\left\|\Delta \Phi_{t}\right\|^{2}+\left\|F^{\prime \prime}(\phi)\right\|_{L^{\infty}}^{2}\left\|\Phi_{t}\right\|^{2}\right) \\
& \quad \leq C\left\|\Phi_{t}\right\|^{2}+C+\frac{\alpha}{4}\left\|\Delta \Phi_{t}\right\|^{2} .
\end{aligned}
$$

So we update $(3.50)$ as

$$
\frac{1}{2} \frac{d}{d t}\|\nabla \mu\|^{2}+\alpha\left\|\nabla \Phi_{t}\right\|^{2} \leq C\left\|\Phi_{t}\right\|^{2}+C+\frac{\alpha}{4}\left\|\Delta \Phi_{t}\right\|^{2} .
$$

Next, we differentiate $(3.1)_{1}$ with respect to $t$ to get

$$
\Phi_{t t}+U_{t} \cdot \nabla \Phi+U \cdot \nabla \Phi_{t}=\Delta \mu_{t} .
$$

Taking the $L^{2}$ inner product of (3.53) with $\Phi_{t}$, in a similar fashion, we have

$$
\begin{aligned}
\frac{1}{2} \frac{d}{d t}\left\|\Phi_{t}\right\|^{2}+\alpha\left\|\Delta \Phi_{t}\right\|^{2} & =\int_{\Omega} F^{\prime}(\phi)_{t} \Delta \Phi_{t} d \mathbf{x}-\int_{\Omega} \Phi_{t}\left(U_{t} \cdot \nabla \Phi\right) d \mathbf{x} \\
& =\int_{\Omega} F^{\prime \prime}(\phi) \Phi_{t} \Delta \Phi_{t} d \mathbf{x}+\int_{\Omega} \Phi\left(U_{t} \cdot \nabla \Phi_{t}\right) d \mathbf{x} \\
\leq & \frac{\alpha}{4}\left\|\Delta \Phi_{t}\right\|^{2}+\frac{1}{\alpha}\left\|F^{\prime \prime}(\phi)\right\|_{L^{\infty}}^{2}\left\|\Phi_{t}\right\|^{2}+\frac{1}{2 \alpha}\|\Phi\|_{L^{\infty}}^{2}\left\|U_{t}\right\|^{2}+\frac{\alpha}{2}\left\|\nabla \Phi_{t}\right\|^{2} \\
\leq & \frac{\alpha}{4}\left\|\Delta \Phi_{t}\right\|^{2}+C\left\|\Phi_{t}\right\|^{2}+C\|\Phi\|_{H^{2}}^{2}\left(\|\nabla \mu\|^{2}+1\right)+\frac{\alpha}{2}\left\|\nabla \Phi_{t}\right\|^{2} \\
\leq & \frac{\alpha}{4}\left\|\Delta \Phi_{t}\right\|^{2}+C\left\|\Phi_{t}\right\|^{2}+C\left(\|\nabla \mu\|^{2}+1\right)+\frac{\alpha}{2}\left\|\nabla \Phi_{t}\right\|^{2},
\end{aligned}
$$

where we have used (3.48). Combining (3.52) and (3.54) we get

$$
\frac{d}{d t}\left(\|\nabla \mu\|^{2}+\left\|\Phi_{t}\right\|^{2}\right)+\alpha\left(\left\|\nabla \Phi_{t}\right\|^{2}+\left\|\Delta \Phi_{t}\right\|^{2}\right) \leq C\left(\left\|\Phi_{t}\right\|^{2}+\|\nabla \mu\|^{2}+1\right) .
$$

Since the RHS of (3.55) is integrable in time according to Lemma 3.2 and Lemma 3.6, upon integrating (3.55) in time we have

$$
\sup _{t \in[0, T]}\left(\|\nabla \mu(\cdot, t)\|^{2}+\left\|\Phi_{t}(\cdot, t)\right\|^{2}\right)+\int_{0}^{T}\left(\left\|\nabla \Phi_{t}(\cdot, \tau)\right\|^{2}+\left\|\Delta \Phi_{t}(\cdot, \tau)\right\|^{2}\right) d t \leq C,
$$

which, together with Lemma 2.5, implies that

$$
\sup _{t \in[0, T]}\left(\|\nabla \mu(\cdot, t)\|^{2}+\left\|\Phi_{t}(\cdot, t)\right\|^{2}\right)+\int_{0}^{T}\left\|\Phi_{t}(\cdot, \tau)\right\|_{H^{2}}^{2} d t \leq C .
$$


From (3.31)-(3.32), (3.34) and (3.56) we then see that

$$
\sup _{t \in[0, T]}\left(\|\mu(\cdot, t)\|_{H^{2}}^{2}+\|\Phi(\cdot, t)\|_{H^{4}}^{2}\right) \leq C .
$$

Now, taking the $L^{2}$ inner product of (3.53) with $\mu_{t}$ we have

$$
\begin{aligned}
\frac{\alpha}{2} \frac{d}{d t}\left\|\nabla \Phi_{t}\right\|^{2}+\left\|\nabla \mu_{t}\right\|^{2} & =-\int_{\Omega}\left(U_{t} \cdot \nabla \Phi+U \cdot \nabla \Phi_{t}\right) \mu_{t} d \mathbf{x}-\int_{\Omega} F^{\prime \prime}(\phi) \Phi_{t} \Phi_{t t} d \mathbf{x} \\
& =\int_{\Omega}\left(U_{t} \Phi+U \Phi_{t}\right) \cdot \nabla \mu_{t} d \mathbf{x}-\int_{\Omega} F^{\prime \prime}(\phi) \Phi_{t} \Phi_{t t} d \mathbf{x} .
\end{aligned}
$$

For the last term on the RHS of (3.58), we have

$$
-\int_{\Omega} F^{\prime \prime}(\phi) \Phi_{t} \Phi_{t t} d \mathbf{x}=-\frac{1}{2} \frac{d}{d t} \int_{\Omega} F^{\prime \prime}(\phi) \Phi_{t}^{2} d \mathbf{x}+\frac{1}{2} \int_{\Omega} F^{\prime \prime \prime}(\phi) \Phi_{t}^{3} d \mathbf{x} .
$$

So we update (3.58) as

$$
\begin{aligned}
\frac{d}{d t}\left(\alpha\left\|\nabla \Phi_{t}\right\|^{2}\right. & \left.+\int_{\Omega} F^{\prime \prime}(\phi) \Phi_{t}^{2} d \mathbf{x}\right)+2\left\|\nabla \mu_{t}\right\|^{2} \\
& =2 \int_{\Omega}\left(U_{t} \Phi+U \Phi_{t}\right) \cdot \nabla \mu_{t} d \mathbf{x}+\int_{\Omega} F^{\prime \prime \prime}(\phi) \Phi_{t}^{3} d \mathbf{x} .
\end{aligned}
$$

We estimate the summand on the RHS of (3.60) as follows: For the first term, we have

$$
\begin{aligned}
& \left|2 \int_{\Omega}\left(U_{t} \Phi+U \Phi_{t}\right) \cdot \nabla \mu_{t} d \mathbf{x}\right| \\
& \quad \leq\left\|\nabla \mu_{t}\right\|^{2}+2\left(\left\|U_{t}\right\|^{2}\|\Phi\|_{L^{\infty}}^{2}+\|U\|_{L^{\infty}}^{2}\left\|\Phi_{t}\right\|^{2}\right) \\
& \quad \leq\left\|\nabla \mu_{t}\right\|^{2}+C\left(\left\|U_{t}\right\|^{2}+1\right) .
\end{aligned}
$$

For the term involving $\Phi_{t}^{3}$, we have

$$
\begin{aligned}
\left|\int_{\Omega} F^{\prime \prime \prime}(\phi) \Phi_{t}^{3} d \mathbf{x}\right| \leq\left\|F^{\prime \prime \prime}(\phi)\right\|_{\infty}\left\|\Phi_{t}\right\|_{L^{3}}^{3} \leq C\left\|\Phi_{t}\right\|_{L^{\infty}}^{2}\left\|\Phi_{t}\right\|_{L^{1}} & \leq C\left\|\Phi_{t}\right\|_{H^{2}}^{2}\left\|\Phi_{t}\right\| \\
& \leq C\left\|\Delta \Phi_{t}\right\|^{2} .
\end{aligned}
$$

Combining (3.60)-(3.62) we have

$$
\frac{d}{d t}\left(\alpha\left\|\nabla \Phi_{t}\right\|^{2}+\int_{\Omega} F^{\prime \prime}(\phi) \Phi_{t}^{2} d \mathbf{x}\right)+\left\|\nabla \mu_{t}\right\|^{2} \leq C\left(\left\|\Delta \Phi_{t}\right\|^{2}+\left\|U_{t}\right\|^{2}+1\right) .
$$

By virtue of Lemma 3.8 and (3.56) we know that the RHS of (3.63) is integrable in time. Therefore, we have

$$
\sup _{t \in[0, T]}\left(\alpha\left\|\nabla \Phi_{t}\right\|^{2}+\int_{\Omega} F^{\prime \prime}(\phi) \Phi_{t}^{2} d \mathbf{x}\right)+\int_{0}^{T}\left\|\nabla \mu_{t}\right\|^{2} d t \leq C,
$$

which, together with (3.56) and $\left(H_{3}\right)$, implies that

$$
\sup _{t \in[0, T]} \alpha\left\|\nabla \Phi_{t}(\cdot, t)\right\|^{2}+\int_{0}^{T}\left\|\nabla \mu_{t}\right\|^{2} d t \leq C+F_{3} \sup _{t \in[0, T]}\left\|\Phi_{t}\right\|^{2} \leq C .
$$

By Poincaré's inequality we then have

$$
\sup _{t \in[0, T]}\left\|\Phi_{t}\right\|_{H^{1}}^{2}+\int_{0}^{T}\left\|\nabla \mu_{t}\right\|^{2} d t \leq C .
$$


Now we derive some consequences of (3.65). First, by Lemma 2.3 and Sobolev embedding we have

$$
\begin{aligned}
\sup _{t \in[0, T]}\|\mu(\cdot, t)\|_{H^{3}} & \leq C \sup _{t \in[0, T]}\left(\|\mu\|+\|\Delta \mu\|_{H^{1}}+\|\nabla \mu\|\right) \\
& \leq C \sup _{t \in[0, T]}\left(\|\mu\|_{H^{1}}+\left\|\Phi_{t}\right\|_{H^{1}}+\|U \cdot \nabla \Phi\|_{H^{1}}\right) \\
& \leq C \sup _{t \in[0, T]}\left(\|\mu\|_{H^{1}}+\left\|\Phi_{t}\right\|_{H^{1}}+\|U\|_{H^{1}}\left(\|\nabla \Phi\|_{L^{\infty}}+\left\|\nabla^{2} \Phi\right\|_{L^{\infty}}\right)\right) \\
& \leq C \sup _{t \in[0, T]}\left(\|\mu\|_{H^{1}}+\left\|\Phi_{t}\right\|_{H^{1}}+\|U\|_{H^{1}}\|\Phi\|_{H^{4}}\right) .
\end{aligned}
$$

Using (3.18) for $\|U\|_{H^{1}}$, (3.57) for $\|\mu\|_{H^{1}}$ and $\|\Phi\|_{H^{4}}$, and (3.65) for $\left\|\Phi_{t}\right\|_{H^{1}}$ we then have

$$
\sup _{t \in[0, T]}\|\mu(\cdot, t)\|_{H^{3}} \leq C .
$$

Second, by Lemma 2.5 we have

$$
\begin{aligned}
\sup _{t \in[0, T]}\|\Phi(\cdot, t)\|_{H^{5}} & \leq C \sup _{t \in[0, T]}\|\Delta \Phi\|_{H^{3}} \\
& \leq C \sup _{t \in[0, T]}\left(\|\mu\|_{H^{3}}+\left\|F^{\prime}(\phi)\right\|_{H^{3}}\right) .
\end{aligned}
$$

By direct calculation and (3.30) we have

$$
\begin{aligned}
\left\|F^{\prime}(\phi)\right\|_{H^{3}} & \leq C\left(1+\|\nabla \Phi\|_{H^{2}}+\|\nabla \Phi\|_{L^{4}}^{2}+\|\nabla \Phi\|_{L^{6}}^{3}+\|\nabla \Phi\|_{L^{4}}\left\|\nabla^{2} \Phi\right\|_{L^{4}}\right) \\
& \leq C\left(1+\|\nabla \Phi\|_{H^{2}}+\|\nabla \Phi\|_{H^{1}}^{2}+\|\nabla \Phi\|_{H^{1}}^{3}+\|\nabla \Phi\|_{H^{1}}\left\|\nabla^{2} \Phi\right\|_{H^{1}}\right),
\end{aligned}
$$

which, together with (3.57), implies that

$$
\sup _{t \in[0, T]}\left\|F^{\prime}(\phi)(\cdot, t)\right\|_{H^{3}} \leq C .
$$

Therefore, combining (3.66)-(3.68) we have

$$
\sup _{t \in[0, T]}\|\Phi(\cdot, t)\|_{H^{5}} \leq C,
$$

which completes the proof.

3.5. $H^{3}$ estimates of $(\Theta, U)$. In this subsection, we shall establish the full regularity of the nonhomogeneous terms on the RHS of the velocity equation in order to prove the regularity of $U$ stated in Theorem 3.1. First, with the help of Lemma 3.9 we have

Lemma 3.10. Under the assumptions of Theorem 1.1, it follows that

$$
\sup _{t \in[0, T]}\|\mu \nabla \Phi(\cdot, t)\|_{H^{3}}^{2} \leq C .
$$

Proof. By direct calculation and Sobolev embedding we have

$$
\begin{aligned}
\|\mu \nabla \Phi\|_{H^{3}}^{2} & \leq C\left(\|\mu\|_{L^{\infty}}^{2}\|\Phi\|_{H^{4}}^{2}+\|\mu\|_{W^{1,4}}^{2}\|\Phi\|_{W^{3,4}}^{2}+\|\mu\|_{H^{2}}^{2}\|\Phi\|_{C^{2}}^{2}+\|\mu\|_{H^{3}}^{2}\|\Phi\|_{C^{1}}^{2}\right) \\
& \leq C\left(\|\mu\|_{H^{2}}^{2}\|\Phi\|_{H^{4}}^{2}+\|\mu\|_{H^{3}}^{2}\|\Phi\|_{H^{3}}^{2},\right.
\end{aligned}
$$

which, together with (3.49), implies (3.70). This completes the proof.

Next, we turn to the $H^{3}$ estimate of $\Theta$ which together with Lemma 3.10 will yield the desired regularity of $U$. 
LEmma 3.11. Under the assumptions of Theorem 1.1, it follows that

$$
\sup _{t \in[0, T]}\|\Theta(\cdot, t)\|_{H^{3}}^{2} \leq C .
$$

Proof. First, we observe that, by (3.48) and Lemma 3.9, it follows that

$$
\sup _{t \in[0, T]}\left\|U_{t}(\cdot, t)\right\|^{2} \leq C .
$$

Second, by taking the temporal derivative of $(3.1)_{4}$ we get

$$
\Theta_{t t}+U_{t} \cdot \nabla \Theta+U \cdot \nabla \Theta_{t}=\kappa \Delta \Theta_{t} .
$$

Taking the $L^{2}$ inner product of (3.73) with $\Theta_{t}$ and using (3.72) we have

$$
\begin{aligned}
\frac{1}{2} \frac{d}{d t}\left\|\Theta_{t}\right\|^{2}+\kappa\left\|\nabla \Theta_{t}\right\|^{2} & =\int_{\Omega} \Theta\left(U_{t} \cdot \nabla \Theta_{t}\right) d \mathbf{x} \\
& \leq \frac{\kappa}{2}\left\|\nabla \Theta_{t}\right\|^{2}+\frac{1}{2 \kappa}\left\|U_{t}\right\|^{2}\|\Theta\|_{L^{\infty}}^{2} \\
& \leq \frac{\kappa}{2}\left\|\nabla \Theta_{t}\right\|^{2}+C\|\Theta\|_{H^{2}}^{2},
\end{aligned}
$$

which gives

$$
\frac{d}{d t}\left\|\Theta_{t}\right\|^{2}+\kappa\left\|\nabla \Theta_{t}\right\|^{2} \leq C\|\Theta\|_{H^{2}}^{2}
$$

Integrating (3.75) in time over $[0, T]$ and using Lemma 3.7 we have

$$
\sup _{t \in[0, T]}\left\|\Theta_{t}\right\|^{2}+\int_{0}^{T}\left\|\nabla \Theta_{t}\right\|^{2} d t \leq C,
$$

which, together with (3.40) and (3.43), implies that

$$
\sup _{t \in[0, T]}\|\Theta\|_{H^{2}}^{2}+\int_{0}^{T}\left\|\Theta_{t}\right\|_{H^{1}}^{2} d t \leq C .
$$

By virtue of Lemma 2.2 with $m=1, p=2$ and (3.76) we have

$$
\begin{aligned}
\|\Theta\|_{H^{3}}^{2} & \leq C\left(\left\|\Theta_{t}\right\|_{H^{1}}^{2}+\|U \cdot \nabla \Theta\|_{H^{1}}^{2}\right) \\
& \leq C\left(\left\|\Theta_{t}\right\|_{H^{1}}^{2}+\|\nabla U\|_{L^{4}}^{2}\|\nabla \Theta\|_{L^{4}}^{2}+\|U\|_{L^{\infty}}^{2}\|\Theta\|_{H^{2}}^{2}\right) \\
& \leq C\left(\left\|\Theta_{t}\right\|_{H^{1}}^{2}+\|\nabla U\|_{L^{4}}^{2}\|\nabla \Theta\|_{H^{1}}^{2}+\|U\|_{L^{\infty}}^{2}\|\Theta\|_{H^{2}}^{2}\right) \\
& \leq C\left(\left\|\Theta_{t}\right\|_{H^{1}}^{2}+1\right),
\end{aligned}
$$

where we used (3.44) and (3.47). Therefore, (3.76)-(3.77) yield

$$
\sup _{t \in[0, T]}\|\Theta(\cdot, t)\|_{H^{2}}^{2}+\int_{0}^{T}\|\Theta(\cdot, t)\|_{H^{3}}^{2} d t \leq C .
$$

We continue to find higher-order estimates for $\Theta_{t}$ in order to improve spatial regularity. To this end, we take the $L^{2}$ inner product of (3.73) with $\Theta_{t t}$ to get

$$
\frac{\kappa}{2} \frac{d}{d t}\left\|\nabla \Theta_{t}\right\|^{2}+\left\|\Theta_{t t}\right\|^{2}=-\int_{\Omega}\left(U_{t} \cdot \nabla \Theta\right) \Theta_{t t} d \mathbf{x}-\int_{\Omega}\left(U \cdot \nabla \Theta_{t}\right) \Theta_{t t} d \mathbf{x} .
$$


By (3.47) and (3.72) we have

$$
\begin{aligned}
& \left|-\int_{\Omega}\left(U_{t} \cdot \nabla \Theta\right) \Theta_{t t} d \mathbf{x}-\int_{\Omega}\left(U \cdot \nabla \Theta_{t}\right) \Theta_{t t} d \mathbf{x}\right| \\
& \quad \leq \frac{1}{4}\left\|\Theta_{t t}\right\|^{2}+\left\|U_{t}\right\|^{2}\|\nabla \Theta\|_{L^{\infty}}^{2}+\frac{1}{4}\left\|\Theta_{t t}\right\|^{2}+\|U\|_{L^{\infty}}^{2}\left\|\nabla \Theta_{t}\right\|^{2} \\
& \quad \leq \frac{1}{2}\left\|\Theta_{t t}\right\|^{2}+C\|\Theta\|_{H^{3}}^{2}+C\left\|\nabla \Theta_{t}\right\|^{2},
\end{aligned}
$$

which, together with (3.79), yields

$$
\kappa \frac{d}{d t}\left\|\nabla \Theta_{t}\right\|^{2}+\left\|\Theta_{t t}\right\|^{2} \leq C\left(\|\Theta\|_{H^{3}}^{2}+\left\|\nabla \Theta_{t}\right\|^{2}\right) .
$$

Upon integrating in time and using (3.76), (3.78) and Poincaré's inequality we have

$$
\sup _{t \in[0, T]}\left\|\Theta_{t}(\cdot, t)\right\|_{H^{1}}^{2}+\int_{0}^{T}\left\|\Theta_{t t}(\cdot, t)\right\|^{2} d t \leq C,
$$

which, together with (3.77), implies that

$$
\sup _{t \in[0, T]}\|\Theta(\cdot, t)\|_{H^{3}}^{2} \leq C .
$$

This completes the proof.

Lemmas 3.10-3.11 give the desired estimates of the nonhomogeneous terms on the RHS of the velocity equation. Let the function $G=\mu \nabla \Phi+\Theta \mathbf{e}_{2}$ in Lemma 2.4. Then it is obvious that $G \in C\left([0, T] ; H^{3}(\Omega)\right)$. So Lemma 2.4 implies

LEMma 3.12. Under the assumptions of Theorem 1.1, it follows that

$$
\sup _{t \in[0, T]}\|U(\cdot, t)\|_{H^{3}}^{2} \leq C .
$$

3.6. Final estimates of the solution. It is clear that, in order to complete the regularity of the solution stated in Theorem 3.1, it remains to show the estimates of $\|\Theta\|_{H^{4}}^{2},\|\mu\|_{H^{5}}^{2}$ and $\|\Phi\|_{H^{7}}^{2}$. These will be done in a quite straightforward way with the help of previously established estimates.

First, since $\left.\Theta\right|_{\partial \Omega}=0$, by Lemma 2.2, Lemma 3.11 and Lemma 3.12 we have

$$
\begin{aligned}
\int_{0}^{T}\|\Theta\|_{H^{4}}^{2} d t & \leq C \int_{0}^{T}\left(\left\|\Theta_{t}\right\|_{H^{2}}^{2}+\|U \cdot \nabla \Theta\|_{H^{2}}^{2}\right) d t \\
& \leq C \int_{0}^{T}\left(\left\|\Theta_{t}\right\|_{H^{2}}^{2}+\|U\|_{H^{3}}^{2}\|\Theta\|_{H^{3}}^{2}\right) d t \\
& \leq C \int_{0}^{T}\left(\left\|\Theta_{t}\right\|_{H^{2}}^{2}+1\right) d t .
\end{aligned}
$$


To deal with $\left\|\Theta_{t}\right\|_{H^{2}}^{2}$, we treat (3.73) as an equation for $\Theta_{t}$. Since $\left.\Theta_{t}\right|_{\partial \Omega}=0$, by Lemma 2.2 we have

$$
\begin{aligned}
\int_{0}^{T}\left\|\Theta_{t}\right\|_{H^{2}}^{2} d t & \leq C \int_{0}^{T}\left(\left\|\Theta_{t t}\right\|^{2}+\left\|U_{t} \cdot \nabla \Theta\right\|^{2}+\left\|U \cdot \nabla \Theta_{t}\right\|^{2}\right) d t \\
& \leq C \int_{0}^{T}\left(\left\|\Theta_{t t}\right\|^{2}+\left\|U_{t}\right\|^{2}\|\nabla \Theta\|_{L^{\infty}}^{2}+\|U\|_{L^{\infty}}^{2}\left\|\nabla \Theta_{t}\right\|^{2}\right) d t \\
& \leq C \int_{0}^{T}\left(\left\|\Theta_{t t}\right\|^{2}+\left\|U_{t}\right\|^{2}\|\nabla \Theta\|_{H^{2}}^{2}+\|U\|_{H^{2}}^{2}\left\|\nabla \Theta_{t}\right\|^{2}\right) d t .
\end{aligned}
$$

Applying (3.72) for $\left\|U_{t}\right\|^{2}$, (3.81) for $\|\nabla \Theta\|_{H^{2}}^{2}$, (3.82) for $\|U\|_{H^{2}}^{2}$, and (3.80) for $\left\|\nabla \Theta_{t}\right\|^{2}$ and $\left\|\Theta_{t t}\right\|^{2}$ we then have

$$
\int_{0}^{T}\left\|\Theta_{t}\right\|_{H^{2}}^{2} d t \leq C
$$

which, together with (3.83), implies that

$$
\int_{0}^{T}\|\Theta\|_{H^{4}}^{2} d t \leq C
$$

Second, by Lemma 2.3, Lemma 2.5 and (3.49), we have

$$
\begin{aligned}
\|\mu\|_{H^{5}}^{2} & \leq C\left(\|\Delta \mu\|_{H^{3}}^{2}+\|\mu\|_{H^{1}}^{2}\right) \\
& \leq C\left(\left\|\Phi_{t}\right\|_{H^{3}}^{2}+\|U \cdot \nabla \Phi\|_{H^{3}}^{2}+1\right) \\
& \leq C\left(\left\|\Delta \Phi_{t}\right\|_{H^{1}}^{2}+\|U \cdot \nabla \Phi\|_{H^{3}}^{2}+1\right) .
\end{aligned}
$$

For the first term on the RHS of (3.85), by $(3.1)_{2}$ we have

$$
\left\|\Delta \Phi_{t}\right\|_{H^{1}}^{2} \leq C\left(\left\|\Delta \Phi_{t}\right\|^{2}+\left\|\nabla \mu_{t}\right\|^{2}+\left\|\nabla\left(F^{\prime}(\phi)_{t}\right)\right\|^{2}\right) .
$$

Using (3.30), Sobolev embedding and (3.49) we have

$$
\begin{aligned}
\left\|\nabla F^{\prime}(\phi)_{t}\right\|^{2} & \leq\left\|F^{\prime \prime \prime}(\phi)\right\|_{L^{\infty}}^{2}\|\nabla \Phi\|_{L^{\infty}}^{2}\left\|\Phi_{t}\right\|^{2}+\left\|F^{\prime \prime}(\phi)\right\|_{L^{\infty}}^{2}\left\|\nabla \Phi_{t}\right\|^{2} \\
& \leq C\|\nabla \Phi\|_{H^{2}}^{2}\left\|\Phi_{t}\right\|^{2}+C\left\|\nabla \Phi_{t}\right\|^{2} \\
& \leq C\left\|\Phi_{t}\right\|_{H^{1}}^{2} .
\end{aligned}
$$

So we update (3.86) as

$$
\left\|\Delta \Phi_{t}\right\|_{H^{1}}^{2} \leq C\left(\left\|\Delta \Phi_{t}\right\|^{2}+\left\|\nabla \mu_{t}\right\|^{2}+\left\|\Phi_{t}\right\|_{H^{1}}^{2}\right),
$$

which, together with (3.56) and (3.65), implies that

$$
\int_{0}^{T}\left\|\Delta \Phi_{t}\right\|_{H^{1}}^{2} d t \leq C
$$

For the second term on the RHS of (3.85), using Lemma 2.1, Lemma 3.9 and Lemma 3.12 we have

$$
\|U \cdot \nabla \Phi\|_{H^{3}}^{2} \leq C\|U\|_{H^{3}}^{2}\|\Phi\|_{H^{4}}^{2} \leq C,
$$

which, together with (3.85) and (3.88), implies that

$$
\int_{0}^{T}\|\mu(\cdot, t)\|_{H^{5}}^{2} d t \leq C .
$$


Third, concerning the estimate of $\|\Phi\|_{L^{2}\left([0, T] ; H^{7}(\Omega)\right)}^{2}$, we have, by Lemma 2.5:

$$
\begin{aligned}
\|\Phi\|_{H^{7}}^{2} & \leq C\|\Delta \Phi\|_{H^{5}}^{2} \\
& \leq C\left(\|\mu\|_{H^{5}}^{2}+\left\|F^{\prime}(\phi)\right\|_{H^{5}}^{2}\right) .
\end{aligned}
$$

From (3.89) we see that it suffices to estimate the second term on the RHS of (3.90). Under the condition $\left(H_{2}\right)$, by direct calculation we have

$$
\left\|F^{\prime}(\phi)\right\|_{H^{5}}^{2} \leq C\left(\|\Phi\|_{H^{5}}^{2}+1\right)
$$

where the constant $C>0$ depends on $\|\phi\|_{C\left([0, T] ; C^{2}(\bar{\Omega})\right)}$ and $F_{1}, F_{2}$ in $\left(H_{2}\right)$. Since $\phi \in$ $C\left([0, T] ; H^{5}(\Omega)\right) \cap C\left([0, T] ; C^{3}(\bar{\Omega})\right)$, we have

$$
\left\|F^{\prime}(\phi)\right\|_{H^{5}}^{2} \leq C
$$

which, together with (3.90) and (3.89), implies that

$$
\int_{0}^{T}\|\Phi(\cdot, t)\|_{H^{7}}^{2} d t \leq C .
$$

Collecting (3.84), (3.89) and (3.91) we have

LEMma 3.13. Under the assumptions of Theorem 1.1, it follows that

$$
\int_{0}^{T}\left(\|\Theta(\cdot, t)\|_{H^{4}}^{2}+\|\mu(\cdot, t)\|_{H^{5}}^{2}+\|\Phi(\cdot, t)\|_{H^{7}}^{2}\right) d t \leq C .
$$

3.7. Exponential decay of $\|\Theta\|_{L^{p}}$. In this last section, we show the exponential decay of the $L^{p}$ norm of $\Theta$ in order to complete the proof of Theorem 3.1.

Lemma 3.14. Under the assumptions of Theorem 1.1, for any $p \in[2, \infty]$, there exists a constant $\eta_{0}(p) \geq 0$ which is independent of $t$ such that for any $t \geq 0$, it follows that

$$
\|\Theta(\cdot, t)\|_{L^{p}} \leq\left\|\Theta_{0}\right\|_{L^{p}} \exp \left\{-\eta_{0}(p) t\right\},
$$

where the constant $\eta_{0}(p)>0$ for $p \in[2, \infty)$ and $\eta_{0}(p)=0$ for $p=\infty$.

Proof. Since the $L^{2}$ version of the lemma has been proved in Lemma 3.1, we shall work on the case of $p \in(2, \infty]$. First, for any $p \in(2, \infty)$, taking the $L^{2}$ inner product of $(3.1)_{4}$ with $|\Theta|^{p-2} \Theta$ we have

$$
\frac{1}{p} \frac{d}{d t}\left(\int_{\Omega}|\Theta|^{p} d \mathbf{x}\right)+(p-1) \kappa \int_{\Omega}|\Theta|^{p-2}|\nabla \Theta|^{2} d \mathbf{x}=0,
$$

which gives

$$
\frac{d}{d t}\left(\int_{\Omega}|\Theta|^{p} d \mathbf{x}\right)+p(p-1) \kappa \int_{\Omega}|\Theta|^{p-2}|\nabla \Theta|^{2} d \mathbf{x}=0 .
$$

Since $\left.\Theta\right|_{\partial \Omega}=0$, so does $g \equiv|\Theta|^{p / 2}$. Applying Poincaré's inequality to $g$ we have

$$
\int_{\Omega}|\Theta|^{p} d \mathbf{x}=\int_{\Omega} g^{2} d \mathbf{x} \leq c_{0} \int_{\Omega}|\nabla g|^{2} d \mathbf{x}=c_{0} \frac{p^{2}}{4} \int_{\Omega}|\Theta|^{p-2}|\nabla \Theta|^{2} d \mathbf{x},
$$

which yields

$$
\frac{4 p(p-1) \kappa}{c_{0} p^{2}} \int_{\Omega}|\Theta|^{p} d \mathbf{x} \leq p(p-1) \kappa \int_{\Omega}|\Theta|^{p-2}|\nabla \Theta|^{2} d \mathbf{x} .
$$


Plugging (3.93) into (3.92) we have

$$
\frac{d}{d t}\left(\int_{\Omega}|\Theta|^{p} d \mathbf{x}\right)+\frac{4(p-1) \kappa}{c_{0} p} \int_{\Omega}|\Theta|^{p} d \mathbf{x} \leq 0,
$$

which yields immediately that

$$
\|\Theta(\cdot, t)\|_{L^{p}}^{p} \leq\left\|\Theta_{0}\right\|_{L^{p}}^{p} \exp \left\{-\frac{4(p-1) \kappa}{c_{0} p} t\right\} .
$$

It follows that

$$
\|\Theta(\cdot, t)\|_{L^{p}} \leq\left\|\Theta_{0}\right\|_{L^{p}} \exp \left\{-\frac{4(p-1) \kappa}{c_{0} p^{2}} t\right\}, \quad \forall p \in(2, \infty) .
$$

We complete the proof by letting $p \rightarrow \infty$.

3.8. Uniqueness. Lemmas 3.2, 3.9 and 3.11-3.14 conclude the regularity and uniform estimates of the solution stated in Theorem 3.1. Therefore, it remains to show the uniqueness of the solution in order to prove Theorem 1.1. We observe that, due to Theorem 3.1 and Sobolev embedding, it follows that $\phi \in C\left([0, T] ; C^{3}(\bar{\Omega})\right) \cap L^{2}\left([0, T] ; C^{5}(\bar{\Omega})\right)$, $\mu \in C\left([0, T] ; C^{1}(\bar{\Omega})\right) \cap L^{2}\left([0, T] ; C^{3}(\bar{\Omega})\right), U \in C\left([0, T] ; C^{1}(\bar{\Omega})\right)$ and $\theta \in C\left([0, T] ; C^{1}(\bar{\Omega})\right) \cap$ $L^{2}\left([0, T] ; C^{2}(\bar{\Omega})\right)$ for any $0<T<\infty$. Therefore, by these estimates, the uniqueness of the solution follows from the standard $L^{2}$ energy estimate. Indeed, by assuming that one has two solutions $\left(\phi_{1}, \mu_{1}, \theta_{1}, U_{1}, P_{1}\right)$ and $\left(\phi_{2}, \mu_{2}, \theta_{2}, U_{2}, P_{2}\right)$, it is straightforward to show that

$$
\begin{aligned}
& \frac{d}{d t}\left(\left\|\phi_{1}-\phi_{2}\right\|^{2}+\left\|\theta_{1}-\theta_{2}\right\|^{2}+\left\|U_{1}-U_{2}\right\|^{2}\right) \\
& \quad \leq C\left(\left\|\phi_{1}-\phi_{2}\right\|^{2}+\left\|\theta_{1}-\theta_{2}\right\|^{2}+\left\|U_{1}-U_{2}\right\|^{2}\right), \quad \forall t \in[0, T],
\end{aligned}
$$

which implies immediately the uniqueness of the solution. We omit the details here.

Theorem 3.2. Under the assumptions of Theorem 1.1, the solution to (1.1), (1.7) is unique.

Theorem 3.1 and Theorem 3.2 conclude our main result, Theorem 1.1. We finish this section with the following remarks.

REMARK 3.1. It is interesting and important to understand the large time asymptotic behavior of the solution to (1.1), (1.7). We note that, using similar arguments in 6, one can show that $\phi$ converges exponentially fast to $\bar{\phi}$ as time goes to infinity, provided that the initial perturbations are sufficiently small. The idea is to first assume $F^{\prime \prime}(\cdot) \geq 0$ on a small neighborhood $I_{\bar{\phi}}=[\bar{\phi}-\delta, \bar{\phi}+\delta]$ and then solve a modified problem with $F$ replaced by an auxiliary function $F_{\bar{\phi}}$ whose second-order derivative is nonnegative on $\mathbb{R}$ and coincides with $F^{\prime \prime}(\cdot)$ on $I_{\bar{\phi}}$. Then, under the smallness assumption on the initial perturbations, it can be shown that the solution to the modified problem is indeed the solution to the original problem and converges exponentially to the constant state as time goes to infinity. The key estimate is (3.15) in this paper. However, the technique does not work without the smallness assumption because in this case the solution to the modified problem does not coincide with the solution to the original problem. We leave the investigation on the asymptotic behavior of the solution with large initial perturbations for the future. 
REMARK 3.2. We observe that, using the arguments in this paper one can show that the results obtained in Theorem 1.1 still hold if either the Dirichlet boundary condition for $\theta$ is replaced by the Neumann boundary condition $\left.\nabla \theta \cdot \mathbf{n}\right|_{\partial \Omega}=0$, or the Neumann boundary conditions for $\phi$ and $\mu$ are replaced by the Dirichlet boundary conditions $\left.\phi\right|_{\partial \Omega}=\tilde{\phi}$ and $\left.\mu\right|_{\partial \Omega}=\tilde{\mu}$, where $\tilde{\phi}$ and $\tilde{\mu}$ are constants and satisfy $\tilde{\mu}=F^{\prime}(\tilde{\phi})$. In the former case, $\theta$ will converge to $\tilde{\theta}=\frac{1}{|\Omega|} \int_{\Omega} \theta_{0}(\mathbf{x}) d \mathbf{x}$ in the $L^{2}$ norm.

REMARK 3.3. The results obtained in Theorem 1.1 can also be generalized to the case of parameter-dependent diffusion coefficients. In other words, one can replace $\Delta \mu$ by $\nabla \cdot(\beta(\phi) \nabla \mu)$ and $\kappa \Delta \theta$ by $\nabla \cdot(\kappa(\phi) \nabla \theta)$ respectively. In this case, the modeling equations describe more realistic phenomena comparing with (1.1). By imposing appropriate conditions on $\beta(\phi)$ and $\kappa(\phi)$ one can study global existence and uniqueness of classical solutions to the more complicated system. The proofs are in the spirit of this paper. We omit the details here.

Acknowledgement. The research of K. Zhao was partially supported by the National Science Foundation through grant DMS 0807406. The author would like to thank the generous support from the National Science Foundation under Agreement No. 0635561. The author would also like to thank his advisor, Professor Ronghua Pan, for support and encouragement.

\section{REFERENCES}

[1] Adams, R. (1975). Sobolev Spaces. New York: Academic Press. MR0450957 (56:9247)

[2] Agmon, S., Douglis, A., Nirenberg, L. (1959; 1964). Estimates near the boundary for solutions of elliptic partial differential equations satisfying general boundary conditions. I \& II. Comm. Pure. Appl. Math. 12: 623-727; 17: 35-92. MR0125307 (23:A2610) MR0162050(28:5252)

[3] Alikakos, N., Bates, P., Fusco, G. (1991). Slow motion for the Cahn-Hilliard equation in one space dimension. J. Differential Equations 90: 81-135. MR1094451 (92a:35152)

[4] Bates, P., Fife, P. (1993). The dynamics of nucleation for the Cahn-Hilliard equation. SIAM J. Appl. Math. 53 no. 4: 990-1008. MR.1232163 (94g:82034)

[5] Bourguignon, J. P., Brezis, H. (1974). Remarks on the Euler equation. J. Funct. Anal. 15: 341-363. MR0344713 (49:9452)

[6] Boyer, F. (1999). Mathematical study of multi-phase flow under shear through order parameter formulation. Asymptotic Analysis 20: 175-212. MR1700669 (2000g:35166)

[7] Boyer, F. (2001). Nonhomogeneous Cahn-Hilliard fluid. Ann. Inst. Henri Poincaré, Anal. non Linéaire 18 no. 2: 225-259. MR1808030(2002d:76032)

[8] Boyer, F. (2002). A theoretical and numerical model for the study of incompressible mixture flows. Computers and Fluids 31 no. 1: 41-68.

[9] Cannon, J., DiBenedetto, E. (1980). The initial value problem for the Boussinesq equations with data in $L^{p}$. Lecture Notes in Math. 771: 129-144. Berlin: Springer. MR565993 (81f:35101)

[10] Carr, J., Gurtin, M., Slemrod, M. (1984). Structured phase transitions on a finite interval. Arch. Rational Mech. Anal. 86: 317-351. MR759767 (86i:80001)

[11] Chae, D. H. (2006). Global regularity for the 2D Boussinesq equations with partial viscosity terms. Adv. Math. 203 no. 2: 497-513. MR2227730(2007e:35223)

[12] Chae, D. H., Imanuvilov, O. (1999). Generic solvability of the axisymmetric 3-D Euler equations and the 2-D Boussinesq equations. J. Differential Equations 156 no. 1: 1-17. MR.1700862 (2000d:76009)

[13] Chella, R., Vinals, J. (1996). Mixing of a two-phase fluid by a cavity flow. Phys. Rev. E 53: 38323840 .

[14] Debussche, A., Dettori, L. (1995). On the Cahn-Hilliard equation with a logarithmic free energy. Nonlinear Analysis 24 no. 10: 1491-1514. MR1327930 (96c:35080) 
[15] Elliott, C., Garcke, H. (1996). On the Cahn-Hilliard equation with degenerate mobility. SIAM J. Math. Anal. 27 no. 2: 404-423. MR.1377481 (97c:35081)

[16] Gunton, J., San Miguel, M., Sahni, P. (1983). in: Domb, Lebowitz (Eds.), Phase transitions and critical phenomena 8. London: Academic Press. MR794319

[17] Gurtin, M., Polignone, D., Vinals, J. (1996). Two-phase binary fluids and immiscible fluids described by an order parameter. Math. Models and Methods in Appl. Sciences 6: 815-831. MR1404829 (99e:76123)

[18] Hmidi, T., Keraani, S. (2007). On the global well-posedness of the two-dimensional Boussinesq system with a zero diffusivity. Adv. Differential Equations 12 no. 4: 461-480. MR2305876 (2009c:35404)

[19] Hou, T., Li, C. (2005). Global well-posedness of the viscous Boussinesq equations. Discr. Cont. Dynam. Sys. 12: 1-12. MR2121245 (2005j:76026)

[20] Kato, T. (1967). On classical solutions of the two-dimensional non-stationary Euler equations. Arch. Ration. Mech. Anal. 25: 188-200. MR0211057 (35:1939)

[21] Ladyzhenskaya, O. A., Solonnikov, V. A., Uraltseva, N. N. (1968). Linear and Quasi-linear Equations of Parabolic Type. AMS.

[22] Lai, M. J., Pan, R. H., Zhao, K. (2008). Initial boundary value problem for 2D viscous Boussinesq equations. Arch. Ration. Mech. Analysis. To appear.

[23] Lions, P. L. (1996). Mathematical Topics in Fluid Mechanics, Vol. I. New York: Oxford University Press. MR 1422251 (98b:76001)

[24] Lions, J. L., Magenes, E. (1961). Problemi ai limiti non omogenei III. Ann. Scuo. Norm. Sup. Pisa XV: 41-101. MR0146526 (26:4048)

[25] Lions, J. L., Magenes, E. (1961). Problèmes aux limites non homogènes IV. Ann. Scuo. Norm. Sup. Pisa XV: 311-326. MR0140938 (25:4351)

[26] Lorca, S., Boldrini, J. (1999). The initial value problem for a generalized Boussinesq model. Nonlinear Analysis 36: 457-480. MR1675260 (99m:35210)

[27] Majda, A., Bertozzi, A. (2002). Vorticity and Incompressible Flow. Cambridge: Cambridge University Press. MR.1867882(2003a:76002)

[28] Miranville, A. (1999). A model of Cahn-Hilliard equation based on a microforce balance. $C$. $R$. Acad. Sci. Paris Sér. I Math. 328 no. 12: 1247-1252. MR.1701394 (2000e:35091)

[29] Onuki, A. (1997). Phase transitions of fluids in shear flow. J. Phys.: Condens. Matter 9: 6119-6157.

[30] Pedlosky, J. (1987). Geophysical Fluid Dynamics. New York: Springer-Verlag.

[31] Wei, J., Winter, M. (1998). Stationary solutions for the Cahn-Hilliard equation. Ann. Inst. Henri Poincaré, Anal. non Linéaire 15 no. 4: 459-492. MR1632937(2000b:35093)

[32] Zhao, K. (2010). 2D inviscid heat conductive Boussinesq equations on a bounded domain. Michigan Math. J. 59: 329-352. MR2677625

[33] Zhao, K. (2009). Initial-boundary value problem for a coupling of Cahn-Hilliard equation with 2D Boussinesq equations. Preprint. 